Review Article

\title{
Current Advances of Polymer Composites for Water Treatment and Desalination
}

\author{
Mohamed R. Berber (iD) ${ }^{1,2}$ \\ ${ }^{1}$ Department of Chemistry, College of Science, Jouf University, Sakaka, Saudi Arabia \\ ${ }^{2}$ Department of Chemistry, Faculty of Science, Tanta University, Tanta 31527, Egypt \\ Correspondence should be addressed to Mohamed R. Berber; mrberber@science.tanta.edu.eg
}

Received 17 December 2019; Revised 12 February 2020; Accepted 17 February 2020; Published 13 April 2020

Academic Editor: James Barker

Copyright (c) 2020 Mohamed R. Berber. This is an open access article distributed under the Creative Commons Attribution License, which permits unrestricted use, distribution, and reproduction in any medium, provided the original work is properly cited.

\begin{abstract}
Over the past five years, a lot of research activities in polymer composites were done in order to improve environmental sustainability and to present advantages for commercial applications of water treatment and desalination. Polymers offered tunable properties, improved processability, remarkable stability, high surface area for fast decontamination, selectivity to eliminate different pollutants, and cost-cutting of water treatment. Hence, the development of polymeric materials is one of the future directions to meet the environmental water standards and to supply the water requirements of the growing populations. This review highlighted the very recent achievements in fabrication, characterization, and applications of polymeric composites used for water treatment and desalination. The polymeric modifications, the addition of functional groups, and the assemblies of nanomaterials were also discussed in detail. In particular, great attention was paid to the recent advances in polymer/polymer composites, polymer/carbon composites, and polymer/clay composites, presenting their usage in the removal of various types of contaminants, e.g., metal ions, dyes, and other toxic pollutants. The review also summarized the main advantages and disadvantages of the different adsorbent materials. Specific attention was paid to the mechanism of adsorption, including chemisorption and physisorption mechanisms. In addition, the challenges and the future perspectives were identified to reach the optimal performance of the different adsorbents.
\end{abstract}

\section{Introduction}

Polymer composites are defined as a combination of polymers with other organic or inorganic materials to provide new material with target properties, like low density, toughness, stiffness, thermal behavior, chemical and mechanical stability, and other specific properties depending on the target of use. A lot of polymer composites were introduced with significant use in water treatment and desalination, including graphene-based composites, carbon-based composites, and clay-based composites [1-3].

The polymer composite research was offered due the need for materials with better adsorption and removal characteristics of metal ions, dyes, and other toxic pollutants. The polymers used for these composites have been mainly classified on the basis of source (natural and synthetic polymers) and also on the basis of structure (linear, branched, and crosslinked polymers). This review describes the source and the structure of these polymers and their composites, properties, and applications in water treatment and desalination as follows [4-8].

\section{Polymer-Polymer Composites}

Polymers are organic materials with a number of excellent characteristics, namely, high mechanical strength, remarkable flexibility, chemical stability, and high surface area. These properties made polymers work as a host for different organic and inorganic materials. Thus, we became able to synthesize different composites with target properties. Hence, polymer composites have attracted much attention for water treatment and desalination. Polymer-polymer composites offered the possibility of tuning the adsorptive properties through blending, crosslinking, and surface 
functionalizing. The main advantages of this category of composites are the simplicity of preparation and applications, the high chemical stability at the harsh operation conditions, the use for the removal of a wide range of pollutants, and the good recyclability with high adsorption capacities. However, on the other hand, the main drawback is still the high cost of production.

2.1. Polymer-Based Adsorbents for Dye Removal. A lot of polymer-polymer composites were synthesized and offered for the purpose of dyes removal from wastewater. For example, Elkady et al. synthesized a copolymer of styrene and acrylonitrile through a solution polymerization process. The obtained copolymer was fabricated to a nanofiber through an electrospinning technique. Then, the surface of this nanofiber was functionalized by the chemical addition of carboxylic acid groups to improve the dyes absorptivity [9]. The scanning electron microscopy (SEM) confirmed the changes of the nanofiber after the functionalization process, indicating a uniform shape for the carboxylated nanofibers compared to the nonfunctionalized nanofibers. These morphological changes affected the adsorption process of dyes from wastewater. Specifically, the adsorption capacity of the basic violet dye reached $67.11 \mathrm{mg} / \mathrm{g}$ in less than $30 \mathrm{~min}$ after the surface modification of the nanofiber.

With no doubt, the low specific surface area and the lack of surface porosity of these two polymers could not be the key factors to achieve such adsorption. Hence, it can be said that the charged groups created on the surface of the copolymer as well as the morphological changes of the nanofiber structure have played important roles to reach such high adsorption value. In this regard, a low-cost, multifunctional, and straw based adsorbent was synthesized by immobilizing ternary carboxylic acid and acrylamide units on straw powders. As reported in this study, the abundant carboxyl and amino groups added to the composite structure have provided a high adsorption capacity of dyes removal. In particular, the adsorption capacities for $\mathrm{MB}$ and MO reached 120.84 and $3053.48 \mathrm{mg} / \mathrm{g}$, respectively, which were 3 and 54 times higher than those of unmodified straw [10].

Cyclodextrin-based composites have also drawn a wide attention as a new generation of adsorbents for dye removal from wastewater due to their extraordinary physicochemical properties and cavities [11-13]. These advanced structural properties of cyclodextrin composites promoted the practical industrial application compared to native cyclodextrin itself [14]. The recent advances of these novel composite materials were reviewed by Zhou et al. [15]. For example, cyclodextrin polymer was crosslinked with polydopamine to prepare an eco-friendly composite for the removal of different dyes. The high adsorption efficiency of these dyes was correlated to the new structural characteristics and the functional groups of the polymer composite [16]. $\beta$-Cyclodextrin immobilized with starch showed similar behavior for dye removal from wastewater [17]. The obtained irregular surface and the functional groups of the $\beta$-cyclodextrinstarch composite (i.e., $\mathrm{COOH}, \mathrm{NH}_{2}$, and $\mathrm{OH}$ ) played significant roles in the dye capture and removal compared to each of $\beta$-cyclodextrin and starch separately. It is worth noting that the pores as well as the functional groups of the composite have improved the interparticle diffusion and the electrostatic interaction of the dyes. Thus, a high adsorption value was attained. In a similar study, an efficient composite for dyes removal was successfully synthesized by grafting cyclodextrin and amino hyperbranched polymer onto cotton fibers. This composite also showed remarkable adsorption properties for dye removal thanks to the created functional groups [18].

In the same direction, cellulose extracted from agrowastes was grafted with the monomers of 2-acrylamido-2methylpropane sulfonic acid and acrylic acid in the presence of a crosslinker to obtain highly efficient polymer composites for dye removal. The SEM image of the obtained polymer composite (Figure 1) showed smooth and long fiber-like threads for untreated cellulose; however, in the case of the crosslinked graft copolymers, flaky and highly thick threads were obtained, indicating surface functionalization of cellulose. The adsorption behavior of this cellulose composite was investigated towards the elimination of cationic (malachite green and crystal violet) and anionic (Congo red; CR) dye from aqueous medium, studying the effect of different adsorption parameters. As discussed, the cationic dyes were removed at $\mathrm{pH} 7.0$ in $90 \mathrm{~min}$, while the anionic dye was adsorbed at $\mathrm{pH}$ of 2.2 in $8 \mathrm{~h}$. The adsorption data fitted well the Langmuir isotherm and the pseudo-second-order model. This study showed that cellulose based copolymers can work as potential adsorbents for uptake of both anionic and cationic dyes from industrial wastewater [19]. Cellulose as a natural polymer was also functionalized with a hyperbranched polyethylenimine for selective removal and separation of different dyes. Specifically, polyethylenimine was covalently linked to the backbone of cellulose molecules through a Schiff-base formation. The $\mathrm{NH}_{2}$ groups of polyethylenimine and the $\mathrm{CHO}$ groups on oxidized-cellulose were linked together, forming a polymer composite. The adsorption behavior of the formed composite was investigated for the elimination of CR and basic yellow dyes in aqueous media. The maximum adsorption capacity of Congo red dye reached $2100 \mathrm{mg} / \mathrm{g}$ and $1860 \mathrm{mg} / \mathrm{g}$ for basic yellow dye. The composite also showed selective adsorption of different dyes, including reactive red and brilliant blue; however, the absorptivity was very low for eosin and bright yellow dyes [20]. It is worthy of note that the hyperbranched structure of the polymer composite worked as a brush, improving the interparticle diffusion of some dye molecules into the composite (depending on the dye size). Thus, a higher adsorption capacity and selectivity is obtained.

In this regard, diethylenetriamine was incorporated with polyacrylonitrile using the electrospinning technique to prepare a composite with a high adsorption efficiency of dyes. The morphology of the prepared composite was investigated by SEM. After the composite formation, the surface of the nanofiber became rougher compared to the untreated fiber (see Figure 2). This roughness was a result of the incorporation of diethylenetriamine units. This chemical modification of the composite structure affected the removal efficiency of the direct dyes. Also, it is important to note that the amount of dye adsorbed had been affected by the ratio of 

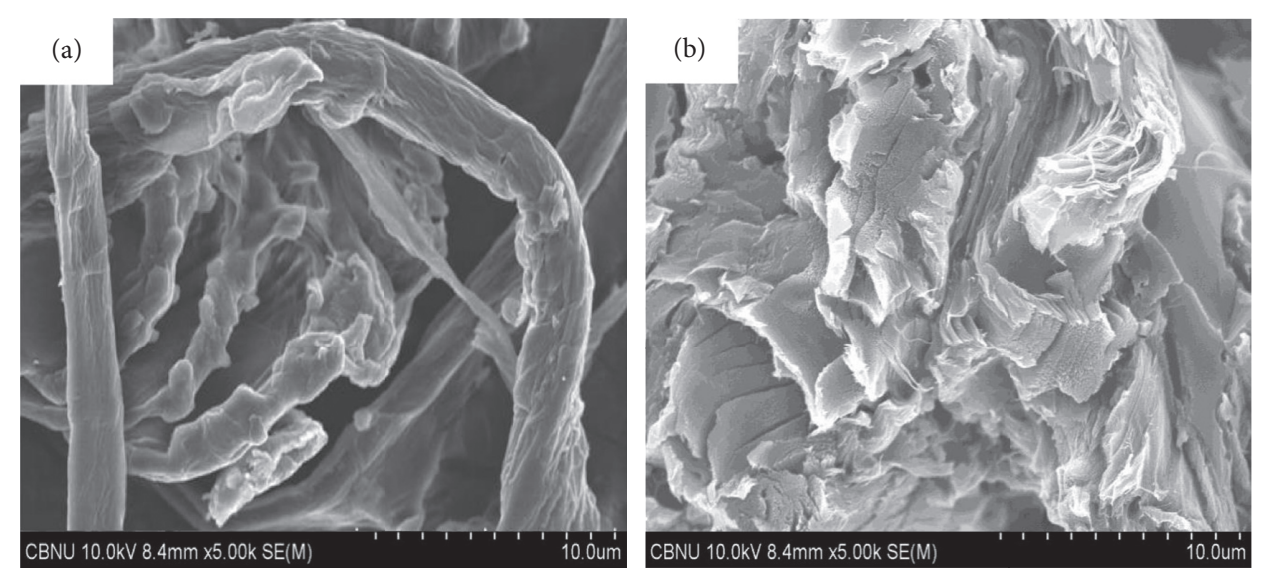

FIGURE 1: SEM images of cellulose (a) before and (b) after copolymerization with 2-acrylamido-2-methylpropane sulfonic acid and acrylic acid (reproduced with a permission from [19]).
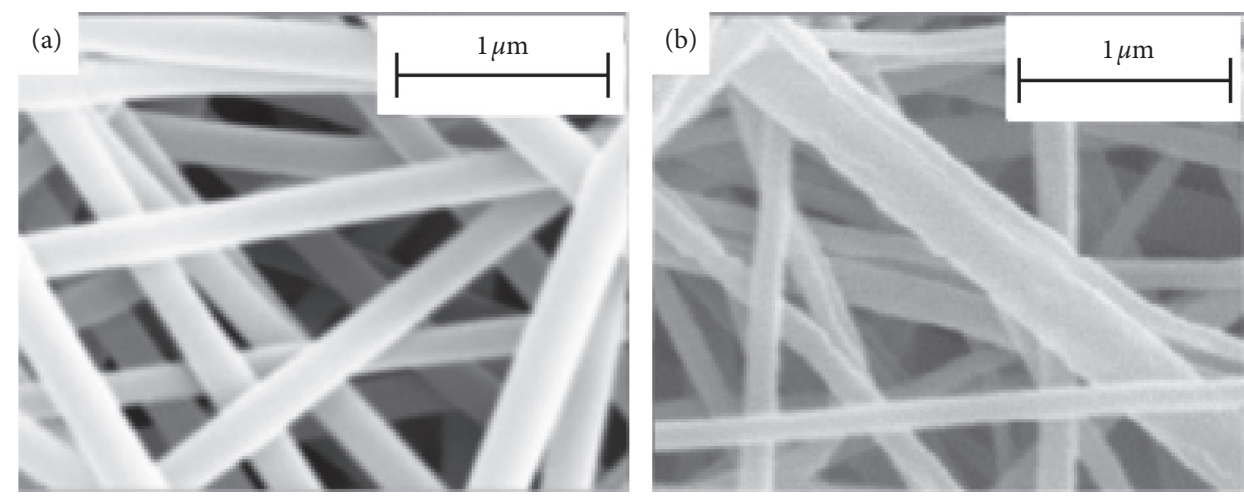

FIGURE 2: SEM images of diethylenetriamine incorporated with polyacrylonitrile (a) before and (b) after surface modification (reproduced with a permission from [21]).

the diethylenetriamine added to the composite, indicating how important the composition ratio is in the composite [21].

\subsection{Polymer-Based Membranes for Desalination and Oil} Removal. Desalination process is a technique for the removal of mineral components from saline water. For that target, many polymer composites were introduced with high potential. For example, a composite membrane of polyvinyl alcohol, cellulose acetate, and polyethylene glycol was prepared and introduced for desalination of groundwater and extremely saline seawater. The values of both water flux and salt rejection were evaluated as a measure of membrane efficiency. The antimicrobial activity was also evaluated for different types of bacteria. The composite membrane showed excellent performance for the desalination of groundwater and the saline water. The flux reduction was reduced significantly when polyethylene glycol was added to the composite structure. The composite structure showed a remarkable performance for antimicrobial activity [22].

In a recent study, a composite membrane was prepared by the addition of p-nitrophenol (PNP) as a plasticizer to cellulose triacetate. The properties of this membrane were tuned to improve its use for desalination as follows. The pristine cellulose triacetate membrane was firstly soaked in a PNP solution and then rinsed with water to obtain the target membrane. This modified membrane showed a reduced salt flux without disclosing the water flux. Accordingly, it showed improved water-salt selectivity [23]. The structural characteristics of cellulose triacetate and modified cellulose triacetate films were evaluated through photo images and polarized optical microscopy images (see Figures 3(a) and 3(b)). Typically, the pristine cellulose triacetate film showed whiteness and opaqueness (Figure 3(a) upper panel), indicating the presence of a crystalline region as confirmed by the birefringence of Figure 3(b) (upper panel). This crystalline structure disappeared in the swelled cellulose triacetate film due to the presence of PNP moieties (Figure 3(a), middle panel). The water rinsing led to the retrieval of the whiteness and the impermeable properties of the cellulose triacetate film and also the birefringence (Figures 3(a) and 3 (b), lower panels), indicating the recrystallization of the cellulose triacetate polymer chains.

The role of PNP treatment in tuning transport properties during the desalination process was illustrated in Figure 4. As seen from image a, untreated cellulose triacetate membrane contained a large crystallite region embedded into an amorphous region. Then when soaked into PNP (Figure $4(\mathrm{~b})$ ), both the crystalline and the amorphous 

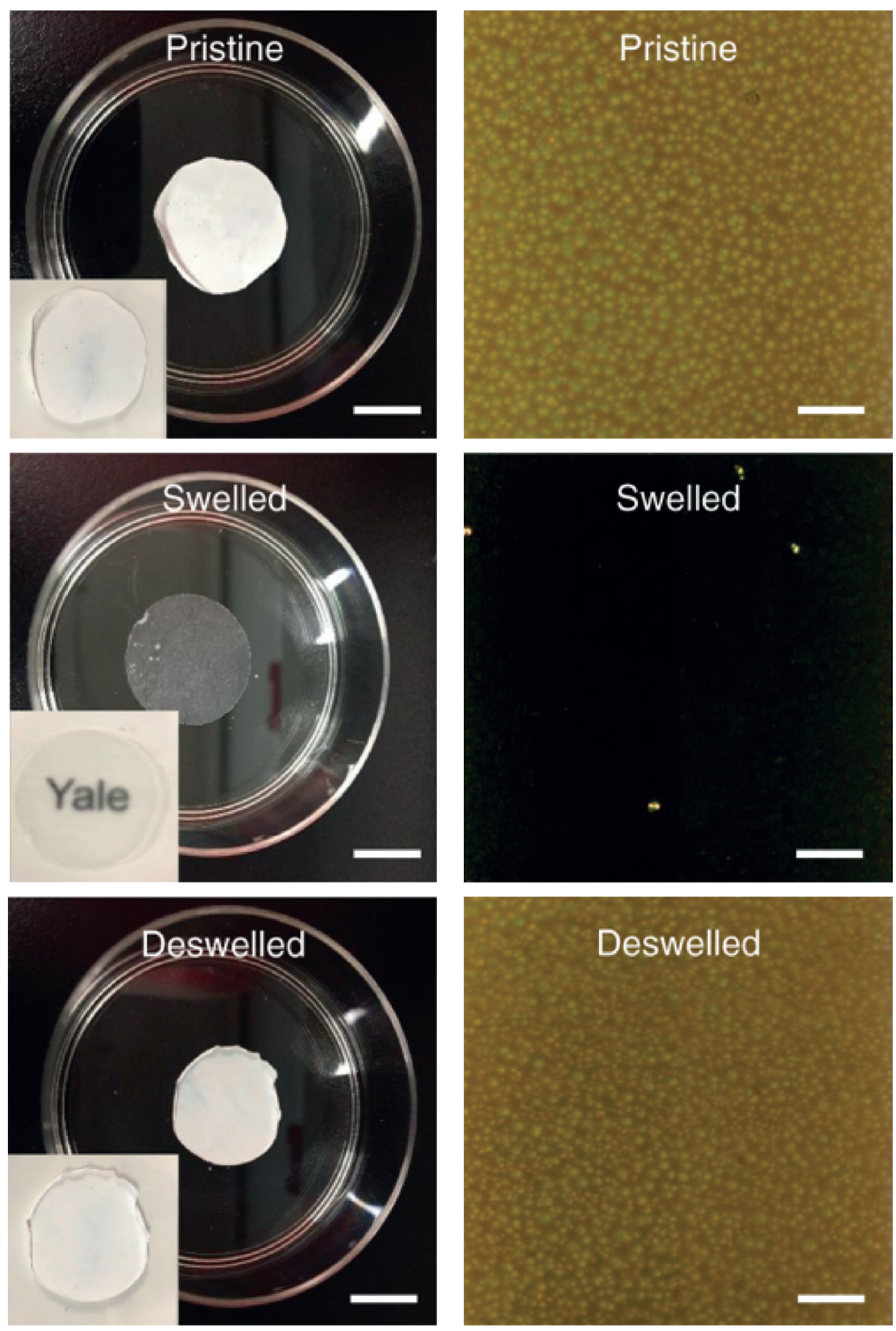

(a)

(b)

Figure 3: (a) Digital photos and (b) polarized optical microscopy images of cellulose triacetate and modified cellulose triacetate films (reproduced with a permission from [23]).

regions swelled where PNP worked as a plasticizer to enhance the chains flexibility. When the polymer matrix was rinsed with water, a rearrangement of the cellulose triacetate chains was obtained, leading to a recrystallization process of the polymer chains (Figure 4(c)). This process formed smaller crystallites; thus, an increase in the amorphous and crystalline regions is obtained. Hence a reduction in the number of nonselective pathways is obtained.

Quaternary ammonium modified polystyrene resins were prepared to improve the oil removal capability of polystyrene. Specifically, the quaternary ammonium surfactants were firstly ion-exchanged with the sulfonic acid groups of polystyrene resin. The formed composite was then grafted with hexadecyl pyridinium, hexadecyltrimethyl ammonium, and tetrabutyl ammonium bromides to change the surface electrostatic and hydrophobic characteristics of the resin. This process made the resin more reactive for the removal the oil droplets by the coalescence filtration process, because a strong hydrophobic and less negative charged resin is obtained [24]. In this regard, Xuanyi Tang et al. prepared a composite of fibric peat modified by hexadecyltrimethylammonium bromide to improve the 


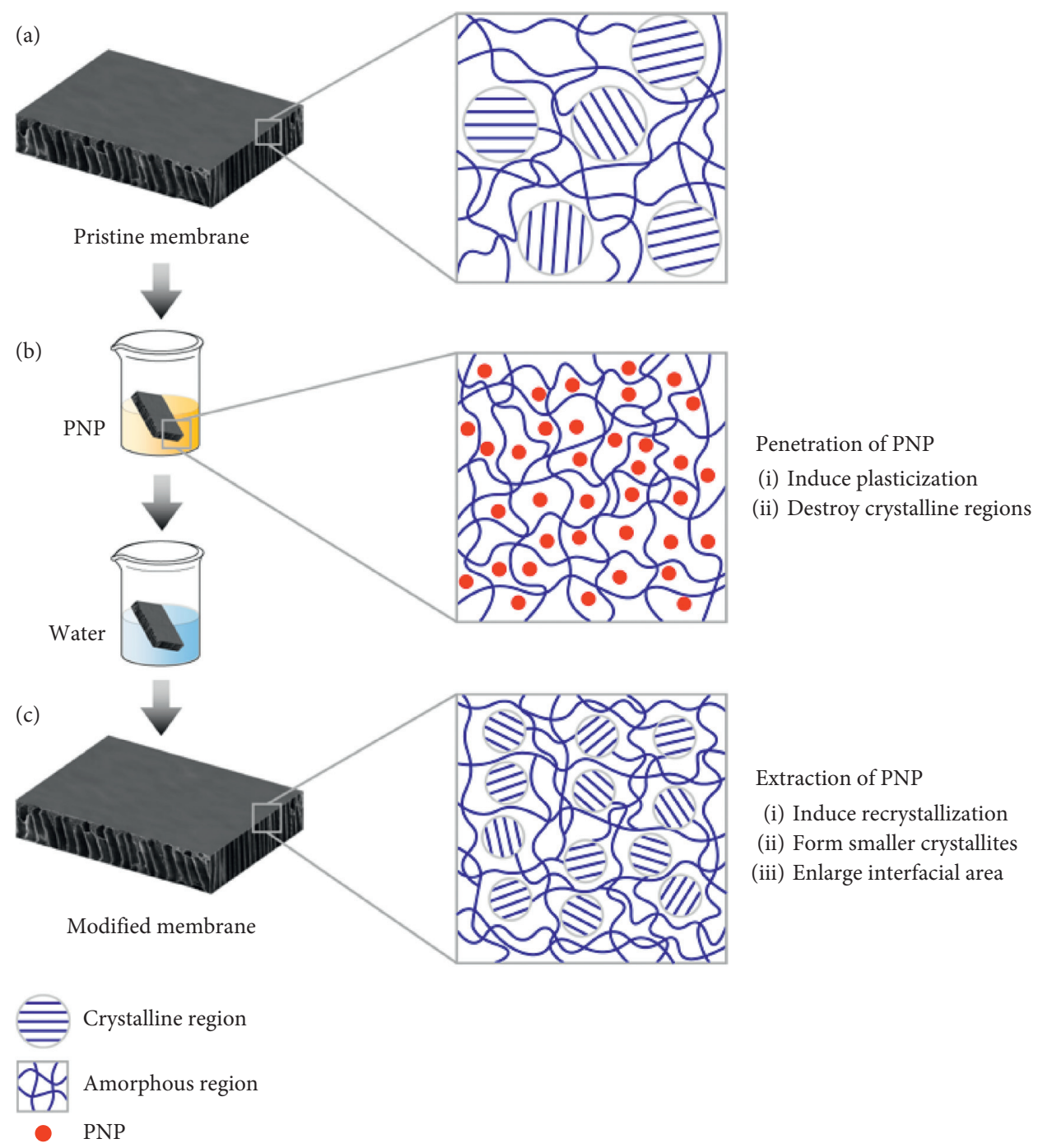

FIgURE 4: A schematic illustration of desalination using PNP treated cellulose triacetate (reproduced with a permission from [23]).

sorption performance of polycyclic aromatic hydrocarbons. It is worth noting that this technique enhanced the hydrophobicity of the fibric peat, leading to enhancement in the sorption rate and capacity of polycyclic aromatic hydrocarbons from wastewater [25].

\subsection{Polymer-Based Adsorbents for Heavy Metals Removal.} The development of the industrial technology has led to the problem of heavy metals which are discharged to the environment through the wastes of painting and electroplating. The adsorption process of heavy metals using low-cost materials is one of the most potential techniques for the removal of heavy metals from wastewater. At present, polymer-polymer composites are receiving a great deal of attention, since they have potential absorptivity, improved stability, and high mechanical feasibility. Copolymerization of polymers is a technique in which two polymers or more are combined together to produce a new material with a target application. For water treatment purposes, the copolymerization process has provided an improvement for the adsorption capacity of single polymers, also improving the polymers aggregation in alkaline medium and polymer disintegration in aqueous solution. The removal mechanism using copolymers is illustrated in Figure 5. The size exclusion mechanism of Figure 5 is the most predominant mechanism of metals removal since the polymer composite membrane worked as a physical barrier. One of the key factors that control the size exclusion mechanism is the pore size of the membrane and the particle size of the target contaminants [26].

Very recently, Checkol et al. [27] showed a polymerpolymer matrix of polystyrene sulfonate/3,4-ethylenedioxythiophene and lignin for the elimination of heavy metals. The polystyrene moieties in the composite made the copolymer work as a cation exchanger, and the sulfonate groups and the lignin moieties enhanced the adsorption capacity of the toxic metals due to the various binding sites on the composite. This study showed that lead ions were adsorbed/desorbed on/from the polymer composite in neutral media when a negative/positive potential was sequentially applied. The adsorption capacity reached 


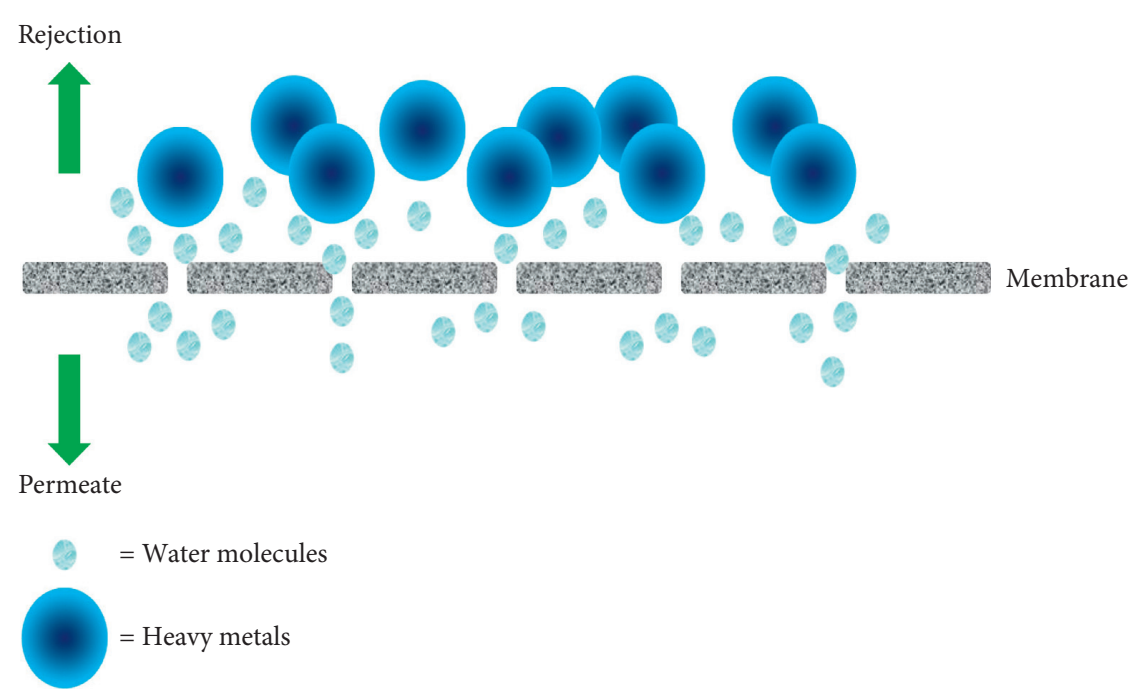

FiguRE 5: Size exclusion/steric hindrance mechanism by low pressure membrane.

$245.5 \mathrm{mg} / \mathrm{g}$ by the polymer-polymer composite and almost doubled $(452.8 \mathrm{mg} / \mathrm{g})$ when lignin moieties were added to the polymer mixture. In this regard, poly(styrene-maleic anhydride) copolymer was modified with cyclopropane and tetramethylenediamine to fabricate a highly adsorbent polymer composite for the elimination of heavy metals. The adsorption selectivity of the metal ions was investigated by the formulated composite at various conditions. The composite showed a high tendency for the removal of some selected metal ions. The affinity order of the composite was copper (II) > zinc (II) > lead (II). The kinetic studies indicated that the adsorption onto the composite followed the pseudo-second-order kinetics and the Langmuir adsorption isotherm [28]. Also, in a recent study, glycidyl methacrylate was grafted with starch. The resultant composite was further coated with polyamine through a cycloaddition between the $\mathrm{NH}_{2}$ of diethylenetriamine and the epoxy groups of glycidyl methacrylate. This polymer composite showed improved adsorption properties towards the heavy metal ions, in particular $\mathrm{Cu}$ and $\mathrm{Pb}$ ions. The adsorption behaviors were explained by a chelation interaction [29].

Porous organic polymers show promising applications in the area of wastewater treatment. Yu et al. introduced a general synthetic route for the porous organic polymer composites which have been used as efficient adsorbents of heavy metals. Specifically, they covalently linked cyclodextrin and EDTA-modified chitosan by pentafluoropyridine to obtain a porous organic polymeric composite using pentafluoropyridine as an environmentally benign solvent. The new polymer composite showed a fast kinetic process for the adsorption of many heavy metals. More importantly, the composite showed five cycles of use with a $91 \%$ adsorption capacity at the fifth cycle. The host-guest inclusion and the chelating effects were the reasons for the high removal efficiency of these polymers [30].

Conducting polymer-based composites are receiving a great deal of attention owing to their potential applications for the elimination of different metal ions. Polypyrrole is a conducting polymer with remarkable properties for the elimination of heavy metals because of its simple preparation process and its biocompatibility properties [31]. Different metal ions were efficiently removed using polypyrrole based composites, in particular, polypyrrole-polyaniline composites, which contained both imine and amine groups that chelated metal ions species through an electrostatic interaction and hydrogen bonding [31].

Understanding how the functional groups of the polymer composite affect the interaction with the heavy metals is of great interest as it will support the future studies to reach the optimum removal efficiency of the target pollutants. For that purpose, Zhang et al. prepared a biosorbent using pine sawdust and citric acid to simultaneously remove the copper ions from wastewater. The study indicated that the functional groups of the composite (hydroxyl and carboxyl) were the reason behind the high adsorption capacity of the copper ions. Specifically, at $\mathrm{pH}>3, \mathrm{COOH}$ groups transformed to $\mathrm{COO}^{-}$groups. Hence, there was an electrostatic interaction between the positive copper ions and the negative charges of the composite. Moreover, the hydroxyl groups contributed to the adsorption of copper ions through a hydrogen bonding network [32].

\section{Polymer-Carbon Composites}

Carbon is an atom with a unique electronic structure, able to form covalent bonds with many other metals and nonmetals. Carbon can exist in different molecular forms. Therefore, a lot of different carbon nanomaterials were synthesized and were used for different applications. Carbon at the nanoscale has many unique properties compared to the other adsorbent materials, namely, high mechanical properties, high electrical conductivity, and high thermal stability. Carbon nanomaterials are classified into different types based on the carbon shape and the geometrical structure. To date, activated carbon, carbon mesosphere, carbon nanotubes, graphite, and graphene are well known carbon nanomaterials and have been used for different applications. The polymer composites of carbon nanomaterials are receiving a 
great deal of attention in different applications, particularly in water treatment and desalination, where the polymer composites of carbon materials possessed excellent properties like high aqueous solubility, large surface area, and enhanced thermal and mechanical stability. These remarkable properties were favorable for the elimination of organic and inorganic pollutants from wastewater. In the following sections, the synthesis, characterization, and water treatment applications of different types of polymer-carbon composites are discussed.

\subsection{Polymer-Based Carbon Nanotubes Composites.} Carbon nanotube (CNT) is a form of the nanocarbon materials with a one-dimension cylindrical nanostructure. CNT possesses an extraordinary graphic nature with a high surface area making it a promising candidate for water treatment and desalination. Despite all these excellent properties, CNTs lack surface functional groups and also possess poor dispersibility in aqueous media. These limitations resulted in a low adsorption performance when CNTs are used as adsorbents. Accordingly, the surface functionalization of CNTs is an important research target in order to improve the water dispersibility and the adsorption performance of CNTs.

3.1.1. For Oil Removal. In a recent study singled-walled $\mathrm{CNTs}$ were added to a copolymer membrane of poly $(\mathrm{N}-$ isopropylacrylamide)-co-(acrylamide) for the separation of water-oil mixture (see Figure 6). CNT is used for that purpose because it has an oleophilic property. The fabricated singled-walled CNTs-based copolymer membrane showed a 99.99\% removal of oil. The photothermal-response property of the singled-walled CNTs-based membrane was noticed to increase the flux of water by light illumination. [33] These research results promoted the applications of CNTs-based polymer for oil removal and stimulated the research groups to find new polymer composites based on CNTs.

In a recent study, multiwalled CNTs were incorporated into a block copolymer of polysulfone/polyether to form a CNTs-polymer membrane which is used to separate oil from water-oil mixture. The study showed the effect of multiwalled-CNTs ratio on the removal efficiency of oil. As noticed, the increase of multiwalled CNTs up to $2.0 \mathrm{wt} \%$ has improved the oil rejection of membrane from $91.4 \%$ to $99.79 \%$. The permeation flux has increased three times when multiwalled-CNTs loading ratio reached $0.5 \mathrm{wt} \%$. The further increase of multiwalled-CNTs loading ratio above $2 \mathrm{wt} \%$ has led to a $30 \%$ decrease in the permeate flux [34]. These studies showed how CNTs are promising candidates for enhancing the removal efficiency of oil from water-oil mixture where CNTs offer self-cleaning and antifouling functions to the polymeric membranes.

3.1.2. For Heavy Metal Removal. Polymer functionalization (physical or chemical) of CNTs is considered as one of the most effective routes to enhance not only the CNTs adsorption properties, but also their hydrophilicity and water solubility. This kind of functionalization showed remarkable electric conductivity and mechanical properties. CNTs were grafted with different polymers to synthesize efficient nanoadsorbents of heavy metals. For example, polyhydroxybutyrate-grafted-CNT composite was synthesized and applied as a nanoadsorbent for the elimination of As, $\mathrm{Cr}$, $\mathrm{Pb}, \mathrm{Cd}, \mathrm{Cu}, \mathrm{Ni}, \mathrm{Zn}$, and $\mathrm{Fe}$ metal ions from industrial wastewater through a batch adsorption process. As proved in this study, the polymer functionalization lowered the degree of agglomeration of CNTs, leading to a significant enhancement in the adsorption characteristic of CNTs. The mechanisms of adsorption by this CNT-polymer composite were ion exchange and electrostatic forces [35].

CNTs coated with polyamidoamine were also synthesized as a nanoadsorbent and applied for the adsorption of Co, $\mathrm{Zn}$, and As. Notably, the adsorption capacities of the studied metal ions were 432, 494, and $470 \mathrm{mg} / \mathrm{g}$ for As, Co, and Zn ions, respectively [36]. In another study, CNTs were incorporated into a polymer hydrogel comprising polyacrylamide and sodium alginate. In this hydrogel, polyacrylamide worked first as flexible network, and then the addition of sodium alginate constructed the rigid network. The addition of CNTs was done to reinforce the mechanical strength and elasticity of the polymer composite structure as well as enhancing the adsorption properties of the polymer composite. This composite exhibited a macroporous structure with a very low density and a high water content, thanks to the addition of CNTs. The adsorption capacity of the fabricated polymer-CNT composite was 1.28 times higher than that of polymer composite only. Figure 7 simply summarizes the synthetic route of this polymer-CNT composite, its morphological structure, and its adsorptive characteristics [37]. Also in another study, polyaniline grafted multiwalled-CNT composite was prepared by an oxidation polymerization process. This composite was then doped with para-toluene-sulfonic acid hydrophilic groups. The addition of such para-toluene-sulfonic acid groups provided the polymer-CNT composite with additional functional groups that were able to enhance the water dispersion of the composite as well as increasing the adsorption characteristics towards chromium ions which are bound to the adsorbent via its amine, imine, and hydroxyl functional groups [38].

3.1.3. For Dye Removal. The composite research of CNTs has attracted much attention to improve CNT-surface characteristics and accordingly enhance its absorptivity of organic pollutants, in particular organic dyes. Surface functionalization of CNTs with polymers is a facile approach to fabricate nanoadsorbent-based CNTs with enhanced adsorption capability of dyes. In a recent study, multiwalled CNTs were grafted with poly(sodium-p-styrene sulfonate). Specifically, CNTs were first coated with dopamine and then self-polymerized to produce polydopamine-CNT composite. The obtained functional polydopamine-CNT composite showed remarkable water dispersibility with enhanced removal of methylene blue dye at various adsorption conditions. The adsorption capacity reached $174 \mathrm{mg} / \mathrm{g}$ after $25 \mathrm{~min}$ 

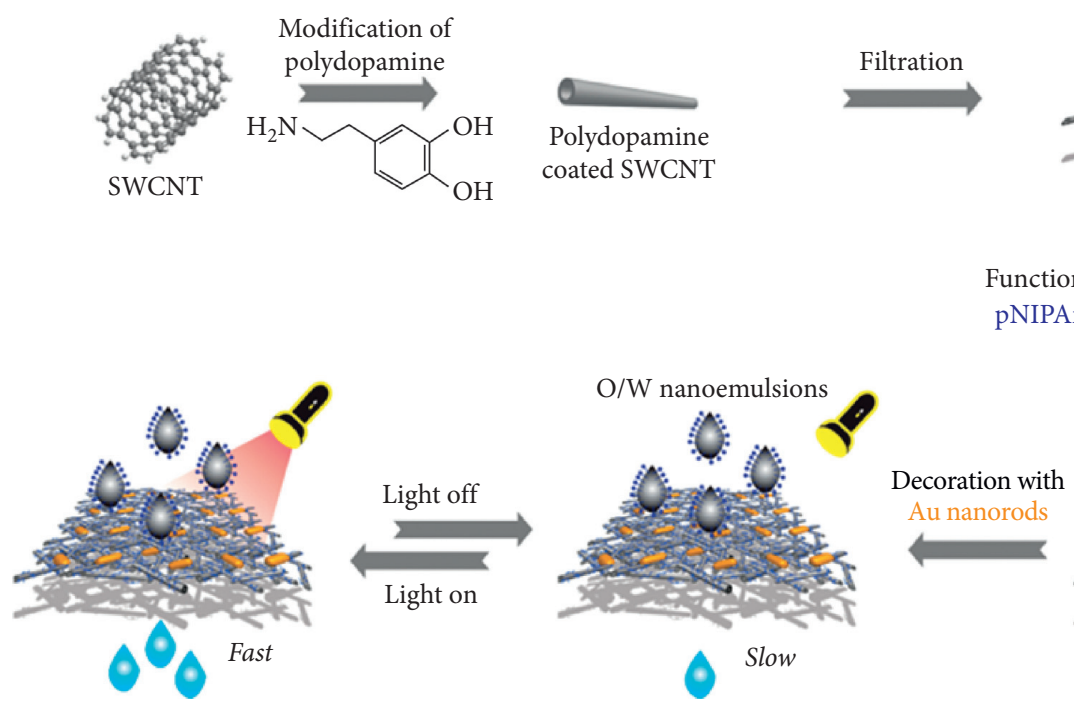

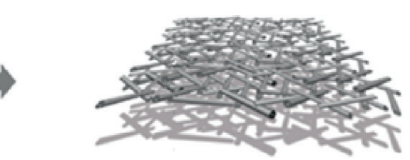

Polydopamine coated SWCNT

Filtration
Functionalization of pNIPAm-co-AAm

FiguRE 6: Schematic illustration of synthesis of singled-walled CNTs incorporated copolymer membrane of poly(N-isopropylacrylamide)co-(acrylamide) and their use in oil removal from water-oil mixture (reproduced with a permission from [33]).
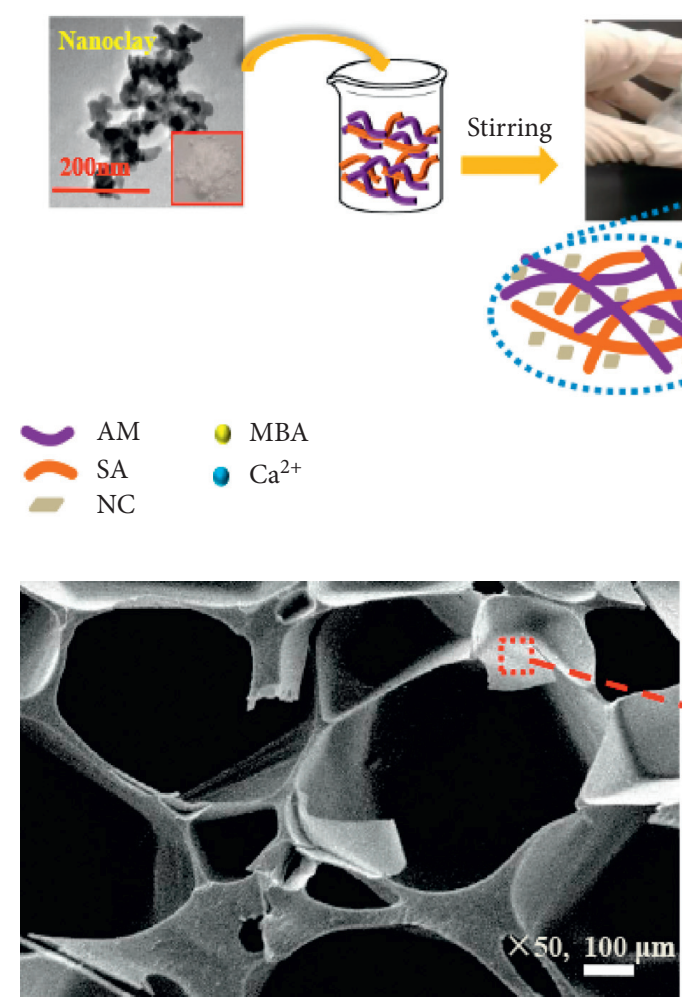

(b)
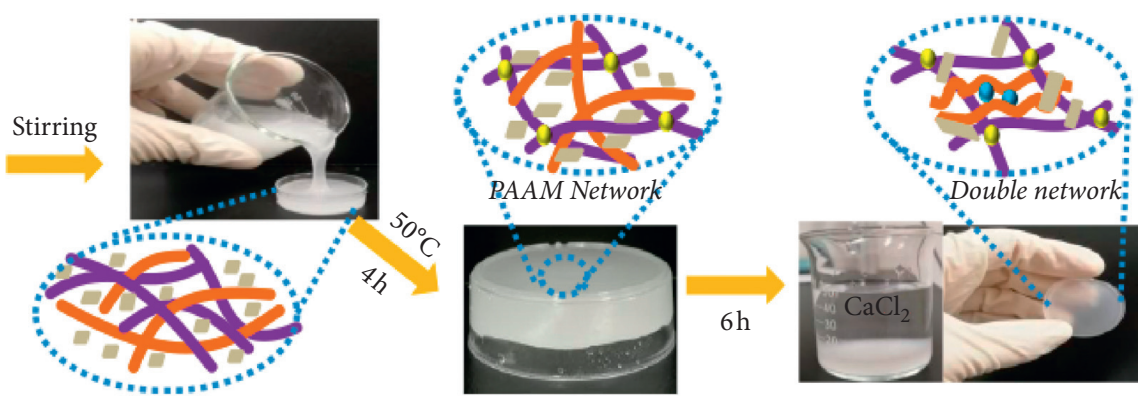

(a)

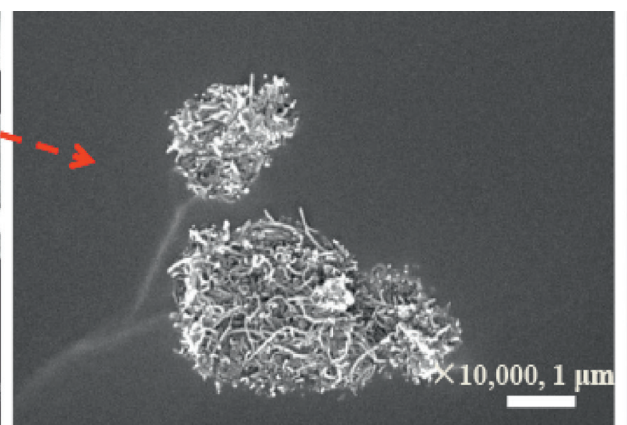

(c)

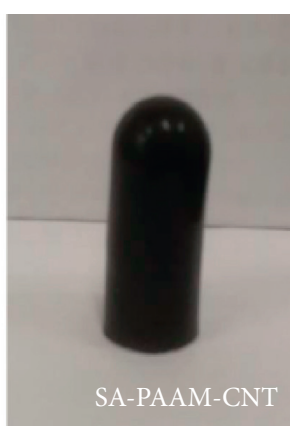

(d)

Figure 7: (a) Schematic illustration of composite formation, (b) SEM image of the freeze-dried polymer network hydrogel, (c) a magnified area of CNT distributed in the polymer matrix, and (d) polyacrylamide-sodium alginate-CNT composite (reproduced with a permission from [37]).

[39]. Another composite based on grafting polyaniline on the surface multiwalled CNTs was recently offered with enhanced adsorptive properties of alizarin yellow dye. The composite was prepared through in situ polymerization of surface oxidized multiwalled CNTs [40]. The maximum adsorption capacity for alizarin yellow dye by this polymerCNT composite was $884.80 \mathrm{mg} / \mathrm{g}$. The data of the adsorption behavior fitted well the pseudo-second-order and the Langmuir isotherm models, respectively. Additionally, the energy changes of the composite showed that the adsorption of alizarin yellow dye was exothermic and spontaneous in nature. Accordingly, this polymer-CNT composite could work as a potential adsorbent for the removal of other organic dyes. 
3.2. Polymer-Graphene Composites. Graphene is a $2 \mathrm{D}$ carbon-based material with a single layer of carbon atoms. Graphene has very interesting physicochemical properties which make it an attractive material for many applications. [41] Graphene-based composite materials are gaining a lot of research interest as nanoadsorbents because such composites possess a three-dimensional structure which provides a higher porosity and a larger specific surface area and aspect ratio, as well as excellent electrical conductivity, mechanical properties, and thermal stability. Hence, a higher absorptivity of organic and inorganic pollutants can be easily obtained. Graphene composites can also be fabricated into membranes for ultrafiltration systems. Therefore, graphenebased composites are excellent candidates for water treatment and desalination.

Polymer-graphene composites are receiving significant importance in water treatment and desalination processes because this kind of composite materials combined the advantages of polymers and graphene, providing a promising adsorbent that can improve the fouling resistance, the selectivity/permeability, the chlorine resistance, and the mechanical stress of the corresponding membranes. Polymer-graphene composites can be synthesized by different techniques depending on the required properties in the target membrane. In the following paragraphs, the preparation techniques as well as the recent water treatment applications of polymer-graphene composites are highlighted.

\subsubsection{Preparation Techniques of Polymer-Graphene Composites}

(1) Spraying Technique. Spraying method is a technique in which a spray-gun is used to spray the polymer-graphene solution onto a preheated substrate. This spraying technique produces a large-area membrane with controllable density. The disadvantage of this technique is the difficulty to obtain a uniform and homogenous membrane.

(2) Vacuum Filtration Technique. The vacuum filtration technique is a two-step process in which graphene is dispersed first and then deposited on a matrix of polymer membrane by vacuum filtration process. This process is very simple and can be used to prepare a uniform polymergraphene membrane.

(3) Spin-Coating Technique. This is a very common and simple technique to prepare a membrane of polymer-graphene composite. Typically, a graphene dispersion is dropped on a rotating-spin coater to fabricate the membrane. By using this technique, a uniform and sizable membrane can be obtained with a controlled thickness.

(4) Layer-by-Layer Self-Assembly Technique. The layer-bylayer self-assembly is one of the most commonly used techniques to fabricate a polymer-graphene composites into membrane. It is an environment-friendly technique and can be used in aqueous media. Please see the details of Figure 8.
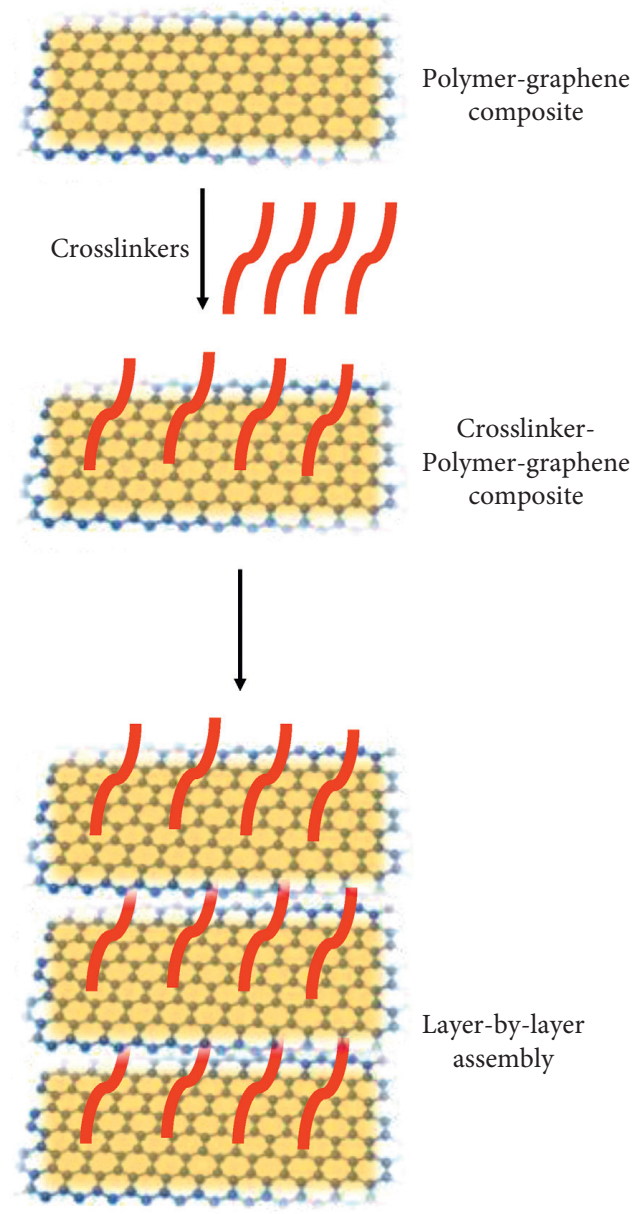

FIGURE 8: A diagrammatic illustration of layer-by-layer self-assembly technique of preparing polymer-graphene composites into membrane.

3.2.2. Applications of Polymer-Graphene Composites for Water Treatment and Desalination. In a recent study, graphene was combined with sodium alginate to prepare a nanocomposite adsorbent. The composite exhibited an enlarged free volume with excellent separation performance and high permeate flux [42]. As illustrated in Figure 9, the hybrid possessed a well-aligned mortar and brick architecture where water channels existed. The structural defects and the free volume cavities of the hybrid have worked for selectivity and permeability of water channels.

In a similar study, poly(N,N-2-ethyl amino ethyl methacrylate) as a temperature-responsive polymer was grafted with graphene oxide in order to prepare a nanomembrane for ultrafiltration. In this composite, poly $(\mathrm{N}, \mathrm{N}-2-$ ethyl amino ethyl methacrylate) induced stimuli to carbon dioxide and argon, producing a controlled pore size into the membrane. The amine group of poly $(\mathrm{N}, \mathrm{N}-2$-ethyl amino ethyl methacrylate) polymer reacted with carbon dioxide in water, protonated, and then deprotonated when argon is fed to the medium. Such change in the morphological shape of the polymer led to a change in separation properties of the cast membrane (see the details of Figure 10). This nanofiltration membrane was tested for the rejection of 


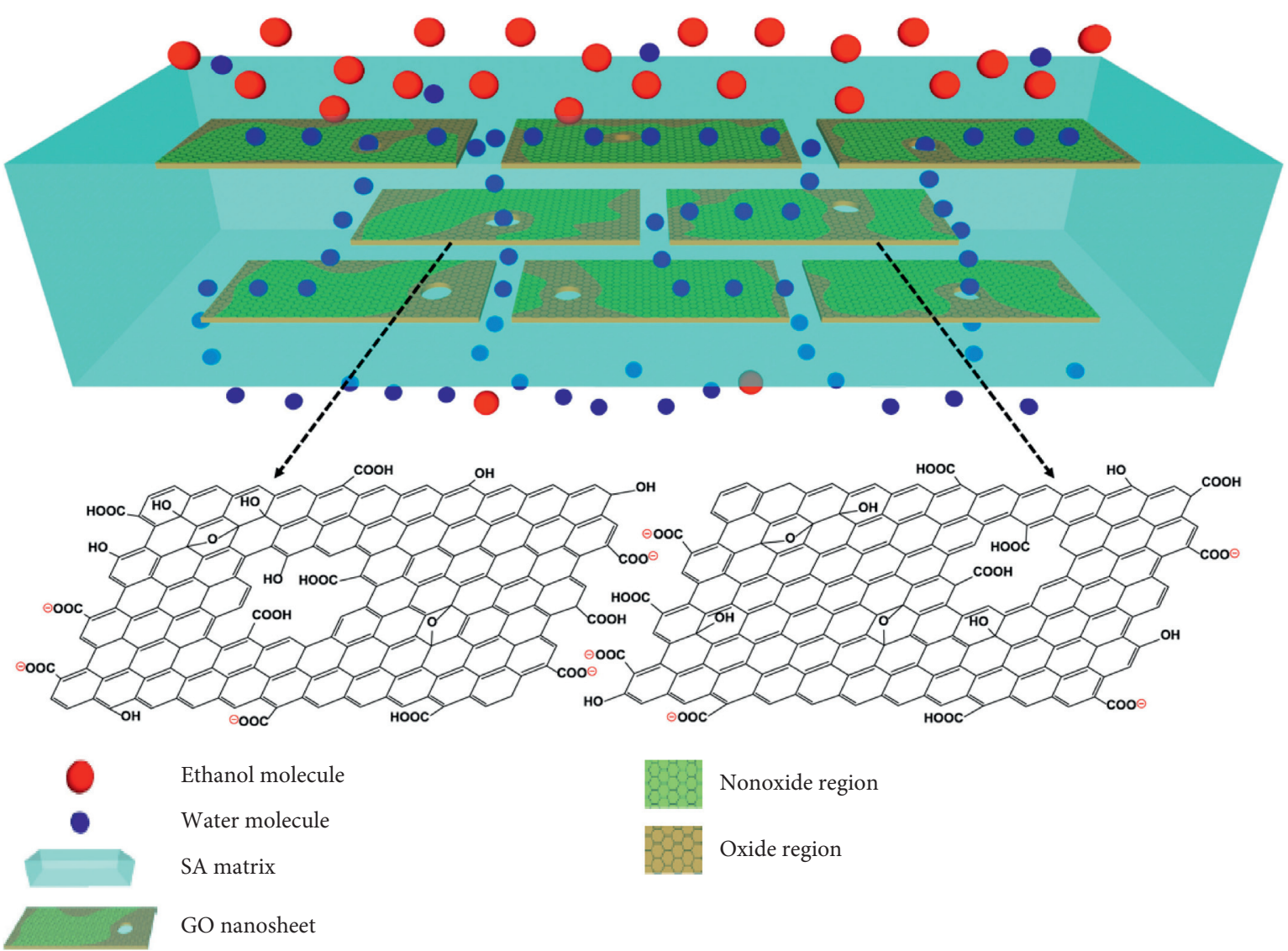

FIGURE 9: A diagrammatic illustration of the mechanism of selective water permeation using graphene-sodium alginate blend (reproduced with a permission from [42]).

rhodamine- $\mathrm{B}$ and the methylene orange dyes. The average rejection rates for these two dyes were 98.9 and 96.5\%, respectively. In addition, the membrane had remarkable repellency to many salts such as magnesium, calcium, and sodium chlorides [43]. For the same target, a crosslinked (Nisopropylacrylamide and $\mathrm{N}, \mathrm{N}$-methylenediacrylamide) polymer was grafted with graphene (see Figure 11). The obtained membrane showed excellent mechanical characteristics with remarkable separation performance and water flux. The water flux of the obtained membrane was $25.8 \mathrm{~L} /$ $\mathrm{m}^{2} \mathrm{~h}$. This value represented three orders of magnitude of the value of the commercial cellulose triacetate membrane [44].

Adsorption capacity, oil-water selectivity, mechanical stability, and reusability at harsh operation conditions are essential parameters for oil-water separation technology. The current state of the art membranes usually sacrifices the mechanical properties and/or recyclability versus membrane performance, i.e., adsorption capacity and selectivity. Thus, it is important to consider all the above-mentioned properties together, in order to offer an efficient membrane for oil-water separation. In a recent study, reduced graphene oxide was grafted polydopamine polymer to fabricate a carbon-based polymer membrane with a light weight and a high separation efficiency of oil from water. This membrane showed an improved chemical stability at harsh operation conditions of acidity and salt concentration. In addition, the separation efficiency of oil reached $99.6 \%$. This remarkable performance was due to the degree of oleophobicity of reduced graphene oxide as well as the superhydrophilicity of polydopamine polymer. The only disadvantage of this membrane was the instability at the alkaline conditions, where the polydopamine polymer dissolved in the alkaline media. [45] In a similar work, a robust and reusable sponge membrane was synthesized by combining poly(dimethylsiloxane) polymer and graphene oxide nanomaterials through a chemical amidation process (see Figure 12). In this polymer-carbon composite, graphene oxide worked as a mechanical fortifier, contributing to the durability of the formed sponge. This composite was tested for the separation of various oils and organic pollutants. The adsorption capacity of the sponge was 724 times its original weight. This study opened the door to develop porous polymer composite membranes with high separation characteristics of oil-water emulsion [46].

To reduce the production cost and to demolish the negative impacts on the environment, cellulose as a natural polymer was applied in the polymer-graphene composites to improve the oil-water separation process. Cellulose showed the advantages of being a low-cost biodegradable material and having high mechanical characteristics. In another study, cellulose-graphene composite membrane was fabricated with ultralight weight and superhydrophobic properties. The obtained aerogel composite showed an excellent adsorption capacity, approximately 80 to 197 times 


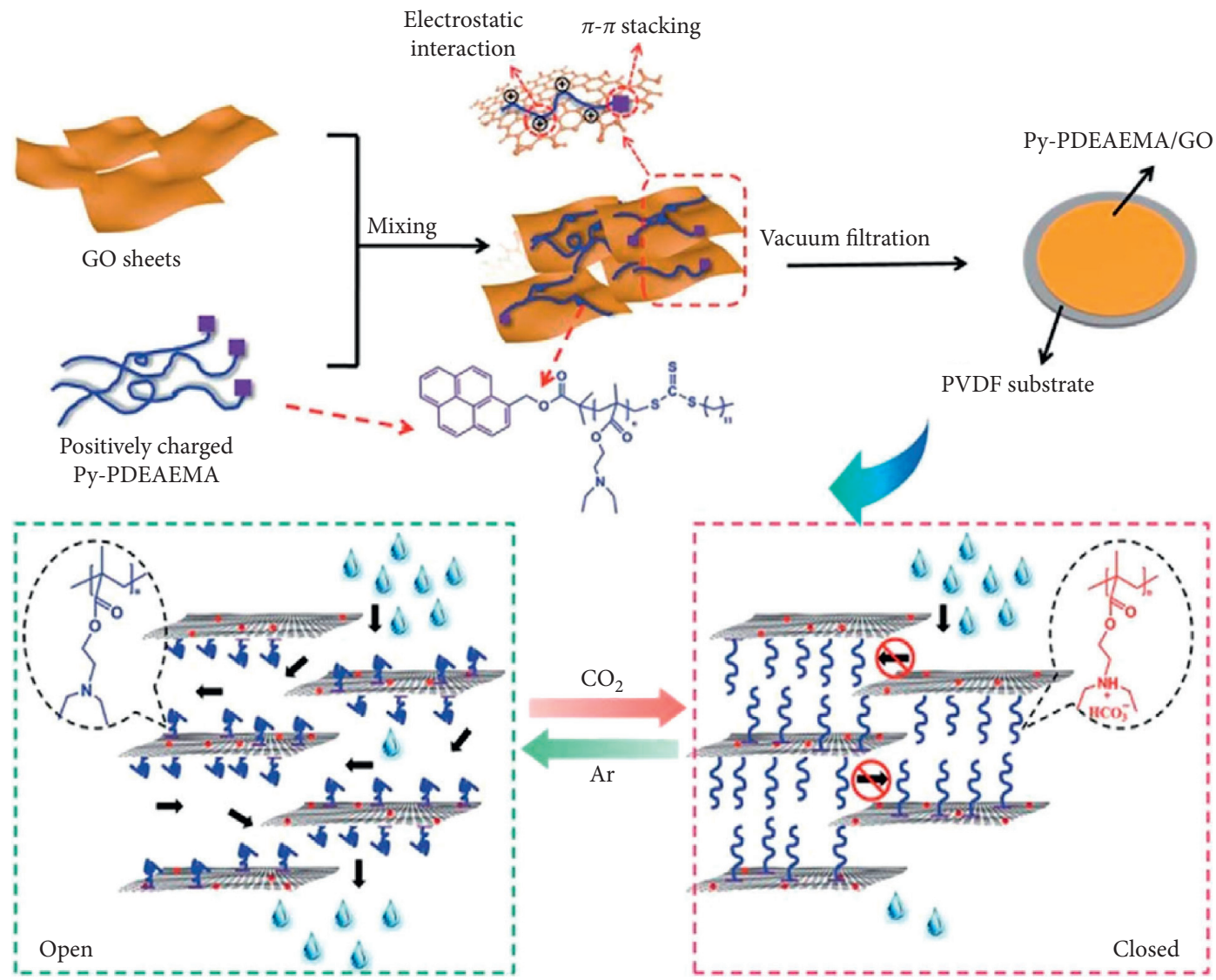

FIGURE 10: Synthetic protocol of poly(N,N-2-ethyl amino ethyl methacrylate)-graphene oxide composite for dyes and salts rejection (reproduced with a permission from [43]).

(oil/organic solvents) its original weight. This performance was obtained due to the multilayered sheet structure of the composite and the large porosity on the composite (see Figure 13). In addition, the composite revealed high mechanical stability even after 100 times of cycling-compression testing [47].

\subsection{Polymer-Based Activated Carbon Composites for Water} Treatment and Desalination. Activated carbon is an amorphous solid carbonaceous material consisting of graphitic lattice. It has many forms including granules, powder, fibers, and cloths. Activated carbon is one of the carbon-based materials used for water treatment and desalination, because it is inexpensive, can be produced from agro-wastes, has a high surface area and porosity. Despite these advantages, some of the activated carbon forms, especially powdered ones, are difficult to remove from the treated aqueous media, because they have very fine powdered forms. Also, they aggregate, leading to a decrease in their adsorptive characteristics. Accordingly, there was a need for new techniques of using activated carbons as potential adsorbents.

The composite formation of activated carbon with polymers has proved to be a promising technique for water treatment and desalination. In a recent study, a composite of cellulose triacetate and activated carbon was prepared by an evaporation/precipitation technique for potential elimination of heavy metals from wastewater.

In this study, the use of a low solvent diffusion process at a low casting temperature demolished the aggregation of activated carbon in the matrix structure. Accordingly, a homogenous membrane was obtained. The addition of activated carbon to cellulose triacetate also supported the production of a less fragile membrane. This cellulose triacetate-activated carbon composite membrane was very efficient for the elimination of arsenic acid from aqueous media, achieving a very high removal value [48]. Polypyrrole as a synthetic polymer was also used to form a composite with activated carbon for the elimination of lead ions from aqueous media. In that work, polypyrrole-activated carbon composite was obtained by a carbonization process of polypyrrole-activated carbon in the presence of a chemical activator. The structural and morphological characterizations supported the composite applicability as an adsorbent. The adsorption capacity of this composite improved with the progress of time and the increase of initial concentration, while it demolished with the increase of adsorbent dose. The adsorption equilibrium of lead ions was described by both Langmuir and Freundlich models. The maximum adsorption reached $50.0 \mathrm{mg} / \mathrm{g}$ after $4 \mathrm{~h}$ at $\mathrm{pH}$ 5.5. The kinetic studies of the composite suggested that the adsorption is a chemisorption process rather than a diffusion process [49]. 


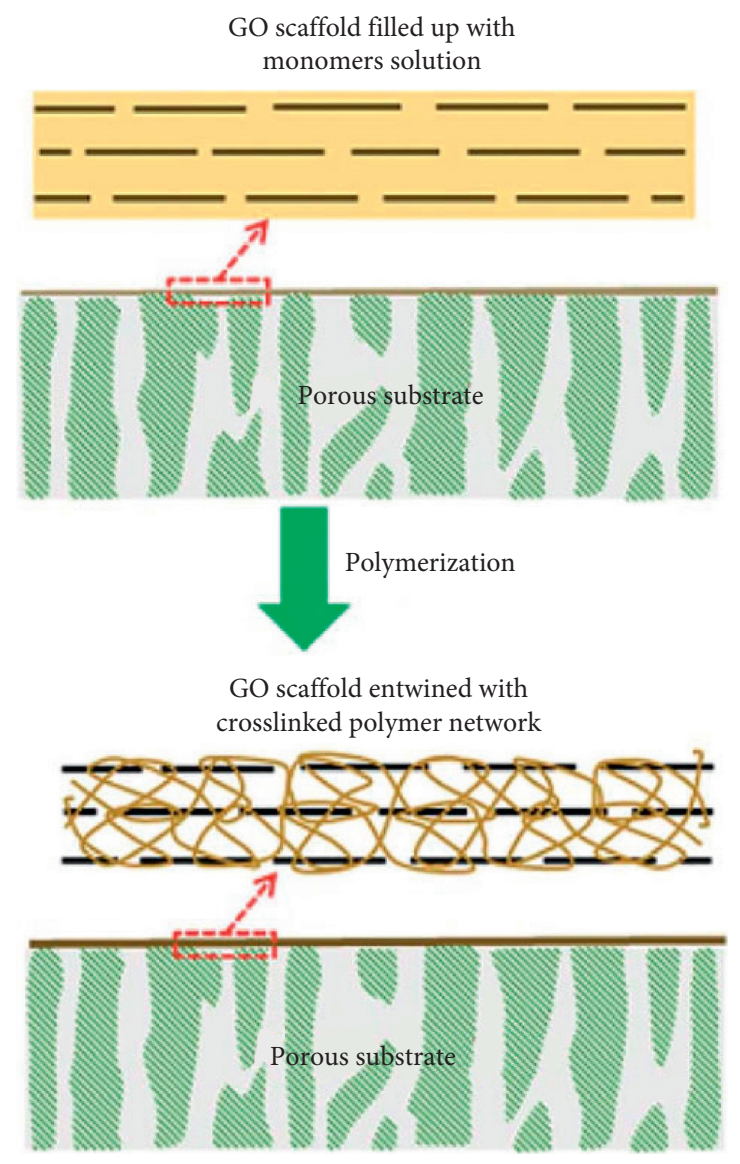

(a)<smiles>C=CC(=O)NCNC(=O)C=C</smiles>

$\mathrm{N}, \mathrm{N}^{\prime}$-methylenebisacrylamide<smiles>C=CC(=O)NC(C)C</smiles>

$\mathrm{N}$-isopropylacrylamide

(b)

Crosslinked polymer network<smiles>CC(C)NC(=O)C(CC(C)(C)C)C(=O)NCNC(=O)C(CC(C)(C)C)C(C)(C)C</smiles>

Poly (N-isopropylacrylamide-co$\mathrm{N}, \mathrm{N}^{\prime}$-methylenebisacrylamide)

(c)

FIGURE 11: Synthetic protocol of crosslinked (N-isopropylacrylamide and N,N-methylenediacrylamide)-graphene composite (reproduced with a permission from [44]).

\section{Polymer-Clay Composites}

Several treatment technologies were explored for the treatment of wastewater sources with the adsorption technique which is a favored process because of its effectiveness, ease of operation, and efficiency. Polymers were used in a composite structure with clays because they can be easily manipulated and produced in a flexible structure. In this section, polymer-clay composites are explored as potential adsorptive materials compared to their individual adsorbents.

Clays are low-cost natural materials, used as adsorbents of many micropollutants. They have a positive or negative layered structure with interlayered anions or cations, respectively. Their use as adsorbents depends on many parameters including porosity, surface area, and particle size. The drawbacks of clays as adsorbents are mainly their $\mathrm{pH}-$ dependence and poor wettability. Thus, clay composite structures were offered to overcome these drawbacks and also to improve their performance in removal selectivity of inorganic and organic contaminants from aqueous media. Clays worked as host matrixes to hold polymers or used as fillers to improve the polymer mechanical properties. Polymer-clay composites attracted attention in water treatment because they exhibited a wide range of pore structures, good surface areas, an ultralight weight gathered with high stiffness, improved processability and stability, good selectivity towards different pollutants, cost effectiveness, and almost no loss on regeneration for reuse. They also offered an easy process for recovering from treated water. Polymer-clay composites can be obtained in different forms by intercalation, coating/wrapping, flocculation, or exfoliation (see Figure 14). In the following paragraphs, the recent polymer-clay composites for water treatment are highlighted.

\subsection{Polymer-Cationic Clay Composites for Water Treatment} and Desalination. A polymer grafted clay composite compiling polyvinylpyridine as a stimuli-responsive polymer and montmorillonite as a cationic clay was synthesized as a novel adsorbent of pollutants with response to $\mathrm{pH}$. The composite structure showed remarkable characteristics, i.e., a high polymer loading without polymer-leaching, a lower $\mathrm{pH} /$ charge dependency, and a high zeta potential. These enhanced properties explained the significant performance towards the elimination of inorganic and organic pollutants, in comparison with the commercial adsorbents. Specifically, at low to moderate $\mathrm{pH}$, the stimuli-responsive polymer accepted protons, leading to an enhancement in the 


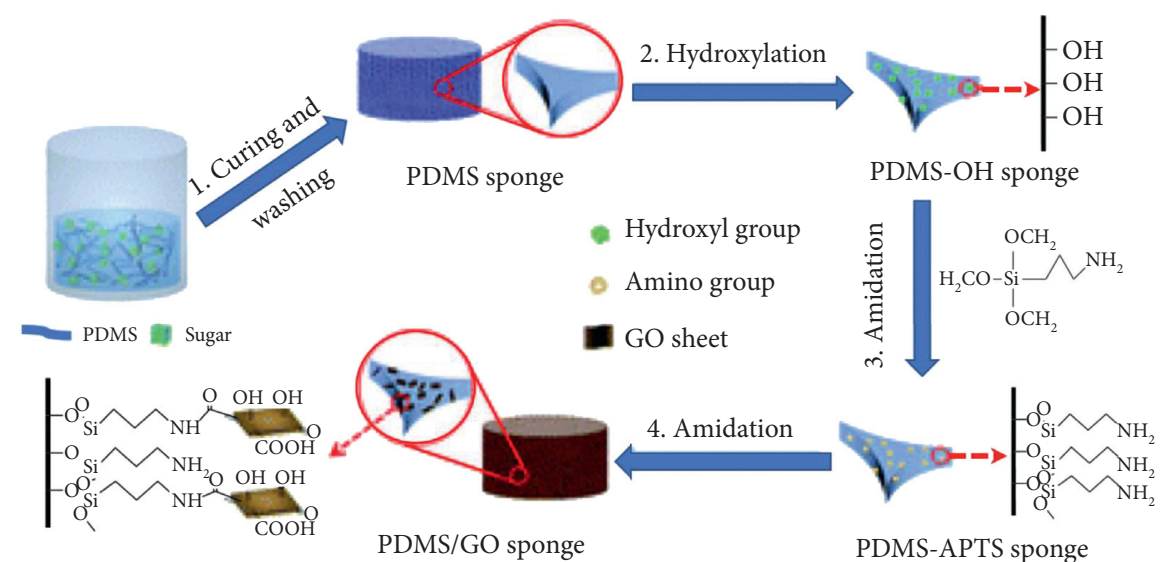

FIgURE 12: A diagrammatic illustration of using a sponge membrane of graphene oxide-grafted poly(dimethylsiloxane) polymer as a separator of oil-water emulsion (reproduced with a permission from [46]).

(a) $\mathrm{CNF} / \mathrm{GO}$ solution
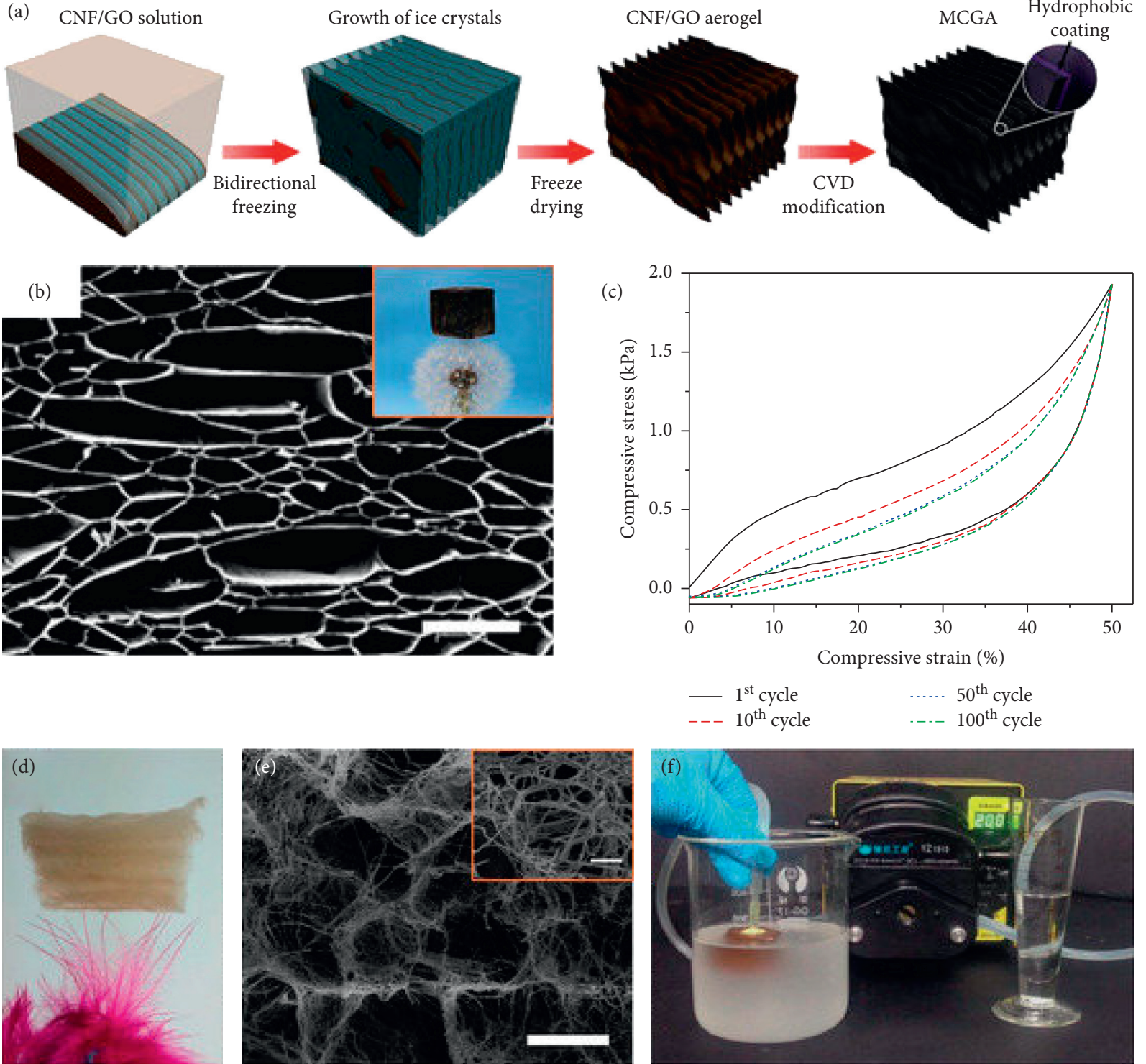

FIGURE 13: A schematic illustration of using cellulose as a low-cost biodegradable material in a polymer-graphene composite for oil-water emulsion separation. The membrane was synthesized by compiling graphene oxide and poly(dimethylsiloxane) polymer (reproduced with a permission from [47]). 


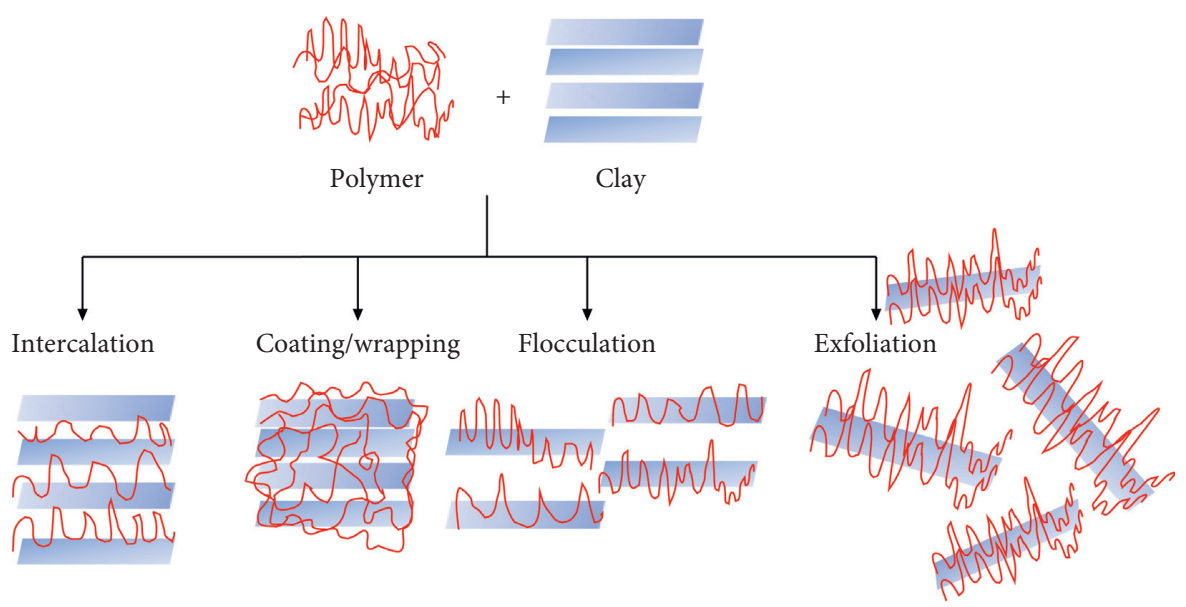

FIGURE 14: A schematic illustration of polymer-clay interaction.

adsorption of the pollutants. However, at elevated $\mathrm{pH}$, the stimuli-responsive polymer lost the protons, leading to desorption of the pollutants. Also, the elimination of micropollutants, e.g., sulfentrazone, arsenate, and atrazine, from wastewater was explored by this composite [50]. In a similar work, exfoliated and intercalated montmorillonites were grafted with different stimuli-responsive polymers, i.e., sodium 2-acrylamido-2-methylpropane sulfonate and $\mathrm{N}$ isopropylacrylamide, using a surfactant-free technique. The prepared composite showed excellent removal capacities towards cobalt, nickel, and methylene blue dye. The results of this composite showed a reduction of surface tension which resulted in improving the absorptivity of the clay, and accordingly an efficient removal of the metal ions and the dye molecules was obtained. This composite also showed reuse of four times with no loss in removal efficiency [51]. In another study, a novel biocomposite was synthesized by incorporating a modified-montmorillonite cationic clay into a polyethylenimine-modified carboxymethyl-chitosan matrix. The composite was prepared to remove the $\mathrm{Hg}$ ions from water. The composite showed a superior removal performance towards $\mathrm{Hg}$ ions from aqueous media. The adsorption capacity reached $1875 \mathrm{mg} / \mathrm{g}$, representing the highest record so far among the literature polymer/clay adsorbents of $\mathrm{Hg}$ ions. This remarkable performance was mainly due to the enhanced porosity of the composite and the increased chelation sites onto the composite structure [52]. For the same target, montmorillonite was complexed with polyvinyl alcohol and poly(4-styrenesulfonic acid-comaleic acid) to offer efficient and cost-effective adsorbents [53].

For dyes removal, a novel hydrogel composite of responsive polymers with montmorillonite was prepared using bis[2-(methacryloyloxy)ethyl] phosphate as a crosslinker. The composite was used for the elimination of methyl red $(\mathrm{MR})$, methylene blue (MB), and crystal violet (CV) from wastewater. Many parameters were applied to obtain a maximum adsorption capacity of the target dyes. Using this composite, the dye removal reached $51 \%$ for $\mathrm{MR}, 89 \%$ for $\mathrm{MB}$, and $80 \%$ for $\mathrm{CV}$ at $\mathrm{pH} 7$ and about $23 \%$ for $\mathrm{MR}, 93 \%$ for $\mathrm{MB}$, and $86 \%$ for $\mathrm{CV}$ at $\mathrm{pH} 12$. The maximum adsorption capacity of this hydrogel composite showed 113,155, and $176 \mathrm{mg} / \mathrm{g}$ for $\mathrm{MR}, \mathrm{MB}$, and $\mathrm{CV}$, respectively. The desorption of the dyes from the composite was simply performed in ethanol solution [54]. For the same target, a hydrogel of acrylamide, $\mathrm{N}$-isopropyl acrylamide, and montmorillonite was synthesized for the elimination of methylene blue (see Figure 15). The prepared composite showed a good swellingdeswelling property. The adsorption process was highly dependent on the $\mathrm{pH}$ and the temperature of the medium. The kinetic studies showed that the adsorption behavior follows the pseudo-second order. The composite also showed a good regeneration capacity with 5 cycles of adsorption-desorption processes. In general, the composite showed a potential use as an environment-friendly adsorbent with efficient absorptivity of dyes from wastewater [55].

\subsection{Polymer-Anionic Clay Composites for Water Treatment} and Desalination. Anionic-clays were also applied as potential adsorbents for water treatment. In a recent study, a novel composite of magnesium-aluminum based layered double hydroxides (LDH) with polydopamine was prepared for the elimination of copper ions from aqueous media (see Figure 16). The results of this study showed a remarkable improvement in the adsorption efficiency of copper ions. The composite showed a two-time higher absorptivity of copper ions compared to pristine layered double hydroxides. The maximum adsorption capacity of copper ions reached $193.78 \mathrm{mg} / \mathrm{g}$ using this composite. The composite was used 4 times with no loss of adsorption capacity. The mechanism of adsorption was due to electrostatic interaction with copper ions [56].

A hydrogel of $\mathrm{Co} / \mathrm{Al}$ layered double hydroxide-polyacrylamide composite was synthesized via in situ polymerization of acrylamide at room temperature in the presence of $\mathrm{LDH}$. The mechanism of hydrogel composite formation was described by coordination and hydrogen bonding interactions between the amide groups of acrylamide and hydroxyl groups of LDH sheets. The composite was utilized for the elimination of methyl orange from wastewater, showing a high adsorption efficiency [57]. 


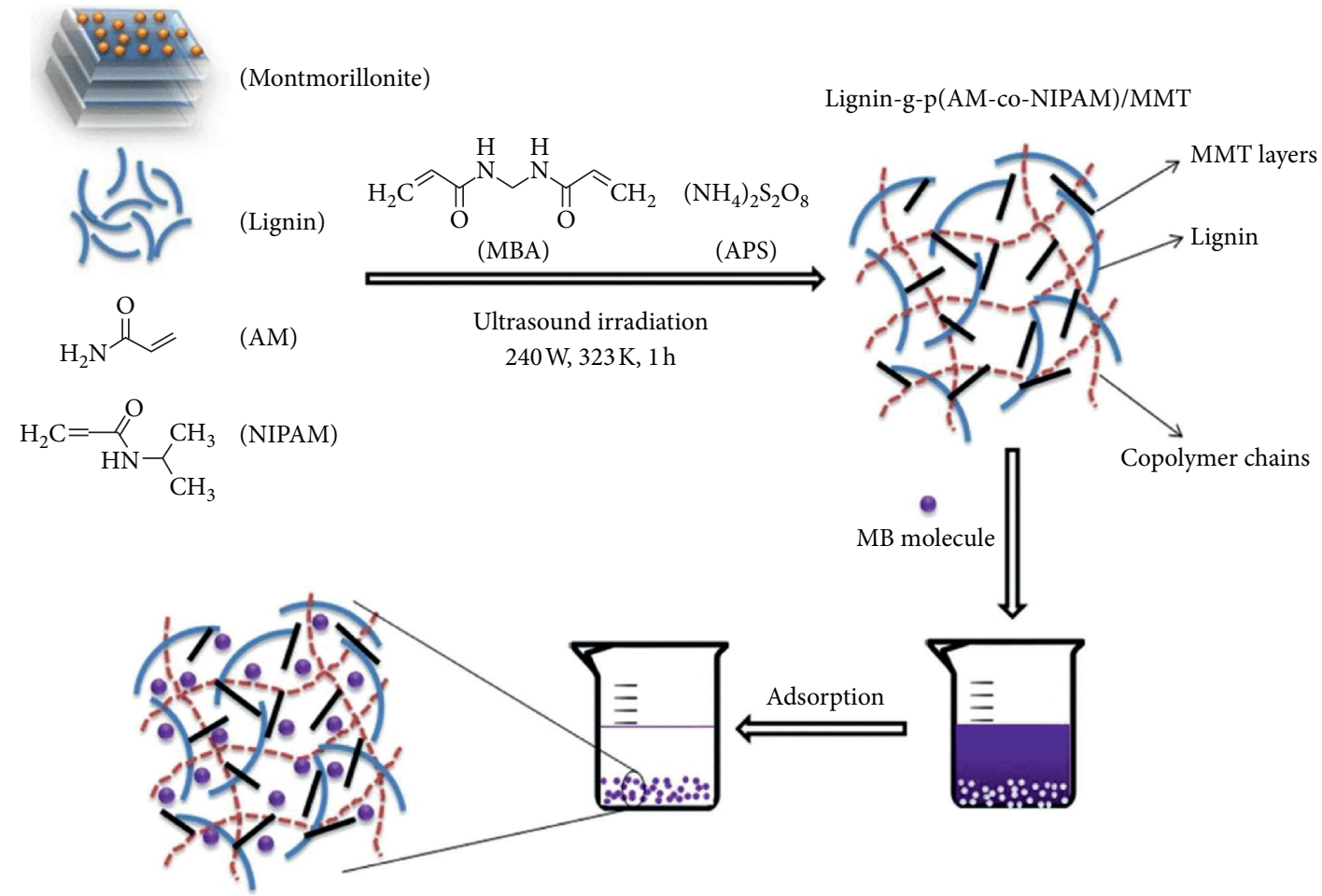

FIgURE 15: A schematic illustration of synthesis of acrylamide and N-isopropyl acrylamide-montmorillonite composite, and its use for methylene blue elimination from aqueous media (reproduced with a permission from [55]).

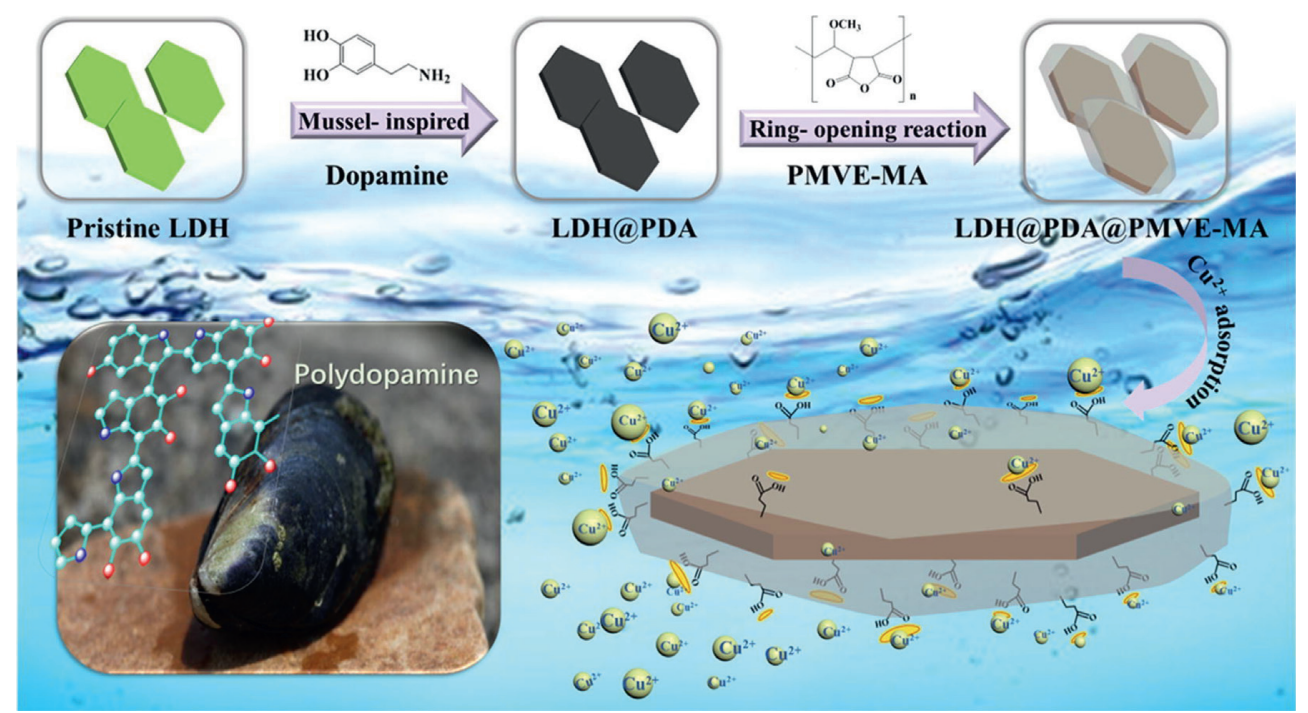

FIGURE 16: A schematic illustration of using polydopamine-LDH composite for elimination of copper ions from wastewater (reproduced with a permission from [56]).

\section{The Mechanisms of Adsorption}

Understanding the mechanism of adsorption is of great importance, since it helps to control and to improve the removal of the different pollutants, as well as increasing the adsorption efficiency. In order to get in-depth insights into the adsorption mechanism, kinetic, isothermal, and thermodynamic studies were performed and applied to the adsorption behavior of the different pollutants. As reported, various adsorption mechanisms have been proposed based on the adsorbent and adsorbate types, sources, structures, and forces of attraction. In this section, the different adsorption mechanisms are highlighted.

5.1. Chemisorption Mechanisms. Chemisorption mechanisms involve the electrostatic interaction mechanism, the ionic exchange mechanism, the reduction/oxidation mechanism, and the complex formation mechanism (chelation) (see Figure 17). These mechanisms can work 


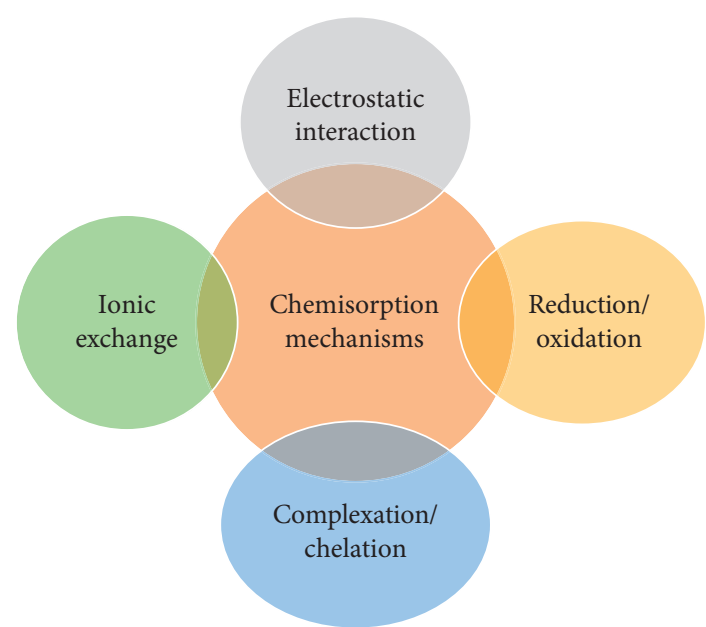

FIgURE 17: Diagrammatic illustration of the different mechanisms involved in chemisorption process.

separately or altogether to remove the pollutants. Specifically, the type of the functional groups present into/onto the adsorbent determines how the adsorption process works (see Figure 18) $[49,58,59]$. For example, if the carboxylic acid groups, the amino groups, or even the hydroxyl groups are present in a protonated form, they undergo a complexation/chelation process to remove the pollutants, but if they are present in a deprotonated form, they undergo an electrostatic interaction process with the pollutants. From the thermodynamic point of view, chemisorption mechanism is usually accompanied by an exothermic process, meaning that energy is liberated during the pollutant adsorption. The enthalpy involved in this process has usually a negative value. This is because of the decrease of the movement of the pollutants when get adsorbed on the surface of the adsorbent. Such process also involves a decrease in the medium entropy because the adsorption is a spontaneous process [60]. The isothermal adsorption studies of the chemisorption mechanism usually show a monolayer formation of the pollutants on the surface of the adsorbent [61]. Furthermore, the chemisorption mechanism may not be detected at low pressure and/or temperature and it became predominant only when the adsorption conditions are changed. From the kinetic point of view, chemisorption mechanism usually follows a pseudo-second-order process which means that the adsorption process usually depends on both the adsorbent and the adsorbate concentrations $[62,63]$.

5.2. Physisorption Mechanisms. Physisorption mechanism occurs between the adsorbates and the adsorbents via Van der Waals forces. Figure 18 shows a schematic illustration of physical adsorption mechanism (lower panel). As indicated, the surface of the adsorbents lacks functionals groups compared to the surface of chemisorption mechanism which contained a lot of functional groups (upper panel). Therefore, the adsorbates tend to physically attach to the adsorbent surface through the weak Van der Waals forces. Physisorption usually occurs at solid surfaces with weak

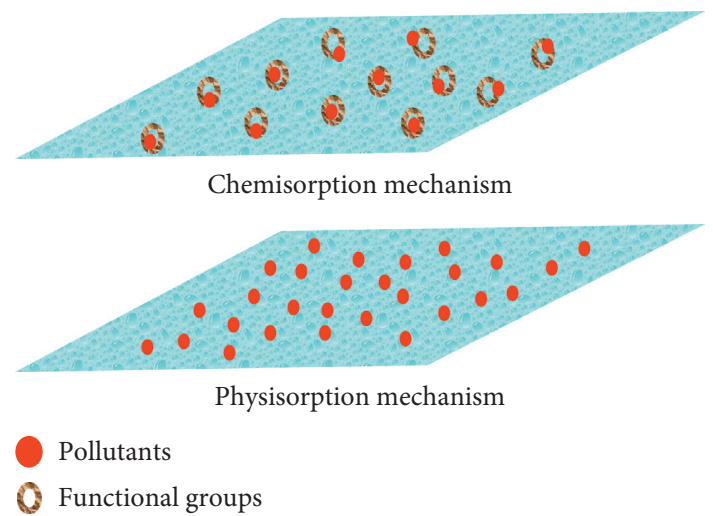

FIgURE 18: Diagrammatic illustration of chemisorption and physisorption mechanism.

binding forces. This mechanism is reversible in nature at a certain extent and is mainly dependent on the pressure and the temperature of the adsorption medium. Thus, the rates of adsorption and desorption are very fast. In the context of kinetics, physisorption mechanism usually follows the pseudo-second order, which means that the adsorption process is mainly dependent on the concentration and the structure of the adsorbents. Hence, porous materials with high surface area are better adsorbents for the physisorption mechanisms. In the context of thermodynamics, physisorption mechanism does not require high activation energy for the adsorption to proceed.

\section{Conclusions and Perspectives}

As shown in this review, both natural and synthetic polymers displayed remarkable adsorptive properties when combined with different adsorbent materials, i.e., carbonbased materials, clays, and other polymers. The polymer composite research opened new research route for a pollution-free environment. Polymers in a composite structure showed a simple and easy fabrication process, a good mechanical performance, and additional binding sites for adsorption of organic pollutants. A lot of research was done to modify the polymer structures to reach high adsorption performance, durability, recyclability, and selectivity of many organic and inorganic pollutants. Despite all the reviewed research articles, polymer composites for water treatment and desalination did not reached their optimal performance especially for the large scale production of such adsorptions. The future perspectives to reach optimal performance of the adsorbents are as follows:

(a) Integrating nanoadsorbent materials with biosorbent carriers in a low cost

(b) Adding/increasing the number of functional groups that are able to enhance the adsorption efficiency, which can be done by exploring new functional materials and/or designing hybrid technologies

(c) Searching for more agro-wastes as adsorbents to decrease the production costs and to sustain the adsorbent-based market 
(d) Studying in detail the mechanism of adsorption to understand the science behind the adsorption behavior and accordingly improve the impacts of the different adsorbents at different conditions

(e) Improving the selectivity of the adsorbents through the selection of the functional groups created on the adsorbents

(f) Improving the pretreatment strategies and processability of the biomass feedstock before being used as adsorbents

(g) Decreasing the operational costs to reach the large scale applications of the adsorbents, which can be done by offering new techniques of pollutants removal

\section{Conflicts of Interest}

The author declares no conflicts of interest.

\section{References}

[1] H. Pang, Y. Wu, X. Wang, B. Hu, and X. Wang, "Recent advances in composites of graphene and layered double hydroxides for water remediation: a review," Chemistry-An Asian Journal, vol. 14, no. 15, pp. 2542-2552, 2019.

[2] X. Liu, R. Ma, X. Wang et al., "Graphene oxide-based materials for efficient removal of heavy metal ions from aqueous solution: a review," Environmental Pollution, vol. 252, pp. 62-73, 2019.

[3] X. Wang, L. Chen, L. Wang et al., "Synthesis of novel nanomaterials and their application in efficient removal of radionuclides," Science China Chemistry, vol. 62, no. 8, pp. 933-967, 2019.

[4] D. Chen, L. Wang, Y. Ma, and W. Yang, "Super-adsorbent material based on functional polymer particles with a multilevel porous structure," NPG Asia Materials, vol. 8, no. 8, p. e301, 2016.

[5] B. Samiey, C.-H. Cheng, and J. Wu, "Organic-inorganic hybrid polymers as adsorbents for removal of heavy metal ions from solutions: a review," Materials, vol. 7 , no. 2, pp. 673-726, 2014.

[6] A. H. Karoyo, J. Yang, and L. D. Wilson, "Cyclodextrin-based polymer-supported bacterium for the adsorption and in-situ biodegradation of phenolic compounds," Frontiers in Chemistry, vol. 6, no. 403, 2018.

[7] H. Guan, D. Zou, H. Yu et al., "Adsorption behavior of iodine by novel covalent organic polymers constructed through heterostructural mixed linkers," Frontiers in Materials, vol. 6, no. 12, 2019.

[8] N. Pandey, S. K. Shukla, and N. B. Singh, "Water purification by polymer nanocomposites: an overview," Nanocomposites, vol. 3, no. 2, pp. 47-66, 2017.

[9] M. Elkady, M. El-Aassar, and H. Hassan, “Adsorption profile of basic dye onto novel fabricated carboxylated functionalized Co-polymer nanofibers," Polymers, vol. 8, no. 5, p. 177, 2016.

[10] Q. Liu, Y. Li, H. Chen et al., "Superior adsorption capacity of functionalised straw adsorbent for dyes and heavy-metal ions," Journal of Hazardous Materials, vol. 382, Article ID 121040, 2020.

[11] Y. Zhou, Y. Hu, W. Huang, G. Cheng, C. Cui, and J. Lu, “A novel amphoteric $\beta$-cyclodextrin-based adsorbent for simultaneous removal of cationic/anionic dyes and bisphenol a," Chemical Engineering Journal, vol. 341, pp. 47-57, 2018.

[12] W. Huang, Y. Hu, Y. Li et al., "Citric acid-crosslinked $\beta$-cyclodextrin for simultaneous removal of bisphenol a, methylene blue and copper: the roles of cavity and surface functional groups," Journal of the Taiwan Institute of Chemical Engineers, vol. 82, pp. 189-197, 2018.

[13] Y. Li, Y. Zhou, Y. Zhou, J. Lei, and S. Pu, "Cyclodextrin modified filter paper for removal of cationic dyes/Cu ions from aqueous solutions," Water Science and Technology, vol. 78, no. 12, pp. 2553-2563, 2019.

[14] Q. Liu, Y. Zhou, J. Lu, and Y. Zhou, "Novel cyclodextrinbased adsorbents for removing pollutants from wastewater: a critical review," Chemosphere, vol. 241, Article ID 125043, 2020.

[15] Y. Zhou, J. Lu, Y. Zhou, and Y. Liu, "Recent advances for dyes removal using novel adsorbents: a review," Environmental Pollution, vol. 252, pp. 352-365, 2019.

[16] H. Chen, Y. Zhou, J. Wang, J. Lu, and Y. Zhou, "Polydopamine modified cyclodextrin polymer as efficient adsorbent for removing cationic dyes and $\mathrm{Cu} 2_{+}$," Journal of Hazardous Materials, vol. 389, Article ID 121897, 2020.

[17] H. Zhang, Y. X. Li, P. L. Wang et al., "Synthesis of $\beta$-cyclodextrin immobilized starch and its application for the removal of dyestuff from waste-water," Journal of Polymers and the Environment, vol. 27, no. 5, pp. 929-941, 2019.

[18] X. Yue, F. Jiang, D. Zhang, H. Lin, and Y. Chen, "Preparation of adsorbent based on cotton fiber for removal of dyes," Fibers and Polymers, vol. 18, no. 11, pp. 2102-2110, 2017.

[19] R. Kumar, R. K. Sharma, and A. P. Singh, "Removal of organic dyes and metal ions by cross-linked graft copolymers of cellulose obtained from the agricultural residue," Journal of Environmental Chemical Engineering, vol. 6, no. 5, pp. 6037-6048, 2018.

[20] W. Zhu, L. Liu, Q. Liao et al., "Functionalization of cellulose with hyperbranched polyethylenimine for selective dye adsorption and separation," Cellulose, vol. 23, no. 6, pp. 37853797, 2016.

[21] A. Almasian, M. E. Olya, and N. M. Mahmoodi, "Preparation and adsorption behavior of diethylenetriamine/polyacrylonitrile composite nanofibers for a direct dye removal," Fibers and Polymers, vol. 16, no. 9, pp. 1925-1934, 2015.

[22] M. A. Ashraf, M. J. Maah, A. K. Qureshi, M. Gharibreza, and I. Yusoff, "Synthetic polymer composite membrane for the desalination of saline water," Desalination and Water Treatment, vol. 51, no. 16-18, pp. 3650-3661, 2013.

[23] X. Lu, X. Feng, Y. Yang et al., "Tuning the permselectivity of polymeric desalination membranes via control of polymer crystallite size," Nature Communications, vol. 10, no. 1, p. 2347, 2019.

[24] Y. Zhou, X. Tang, Y. Xu, and J. Lu, "Effect of quaternary ammonium surfactant modification on oil removal capability of polystyrene resin," Separation and Purification Technology, vol. 75, no. 3, pp. 266-272, 2010.

[25] X. Tang, Y. Zhou, Y. Xu, Q. Zhao, X. Zhou, and J. Lu, "Sorption of polycyclic aromatic hydrocarbons from aqueous solution by hexadecyltrimethylammonium bromide modified fibric peat," Journal of Chemical Technology \& Biotechnology, vol. 85, no. 8, pp. 1084-1091, 2010.

[26] N. Abdullah, N. Yusof, W. J. Lau, J. Jaafar, and A. F. Ismail, "Recent trends of heavy metal removal from water/wastewater by membrane technologies," Journal of Industrial and Engineering Chemistry, vol. 76, pp. 17-38, 2019. 
[27] F. Checkol, A. Elfwing, G. Greczynski, S. Mehretie, O. Inganäs, and S. Admassie, "Highly stable and efficient lignin-PEDOT/PSS composites for removal of toxic metals," Advanced Sustainable Systems, vol. 2, no. 1, Article ID 1700114, 2018.

[28] M. Hosseinzadeh, "Removal of heavy metal ions from aqueous solutions using modified poly(styrene-alt-maleic anhydride) copolymer as a chelating resin," Russian Journal of Applied Chemistry, vol. 91, no. 12, pp. 1984-1993, 2018.

[29] Y. Chen, W. Zhao, H. Wang, X. Meng, and L. Zhang, "A novel polyamine-type starch/glycidyl methacrylate copolymer for adsorption of $\mathrm{Pb}$ (II), $\mathrm{Cu}$ (II), Cd (II) and Cr (III) ions from aqueous solutions," Royal Society Open Science, vol. 5, no. 6, Article ID 180281, 2018.

[30] T. Yu, Z. Xue, X. Zhao, W. Chen, and T. Mu, "Green synthesis of porous $\beta$-cyclodextrin polymers for rapid and efficient removal of organic pollutants and heavy metal ions from water," New Journal of Chemistry, vol. 42, no. 19, pp. 16154-16161, 2018.

[31] H. N. Muhammad Ekramul Mahmud, A. K. O. Huq, and R. B. Yahya, "The removal of heavy metal ions from wastewater/aqueous solution using polypyrrole-based adsorbents: a review," RSC Advances, vol. 6, no. 18, pp. 14778-14791, 2016.

[32] R. Zhang, Y. Zhou, X. Gu, and J. Lu, "Competitive adsorption of methylene blue and $\mathrm{Cu} 2_{+}$onto citric acid modified pine sawdust," CLEAN-Soil, Air, Water, vol. 43, no. 1, pp. 96-103, 2015.

[33] L. Hu, S. Gao, X. Ding et al., "Photothermal-responsive singlewalled carbon nanotube-based ultrathin membranes for on/ off switchable separation of oil-in-water nanoemulsions," ACS Nano, vol. 9, no. 5, pp. 4835-4842, 2015.

[34] J. Saadati and M. Pakizeh, "Separation of oil/water emulsion using a new PSf/pebax/F-MWCNT nanocomposite membrane," Journal of the Taiwan Institute of Chemical Engineers, vol. 71, pp. 265-276, 2017.

[35] M. T. Bankole, A. S. Abdulkareem, I. A. Mohammed et al., "Selected heavy metals removal from electroplating wastewater by purified and polyhydroxylbutyrate functionalized carbon nanotubes adsorbents," Scientific Reports, vol. 9, no. 1, p. 4475, 2019.

[36] B. Hayati, A. Maleki, F. Najafi et al., "Heavy metal adsorption using PAMAM/CNT nanocomposite from aqueous solution in batch and continuous fixed bed systems," Chemical Engineering Journal, vol. 346, pp. 258-270, 2018.

[37] Y. Yue, X. Wang, Q. Wu, J. Han, and J. Jiang, “Assembly of polyacrylamide-sodium alginate-based organic-inorganic hydrogel with mechanical and adsorption properties," Polymers, vol. 11, no. 8, p. 1239, 2019.

[38] R. Kumar, M. O. Ansari, A. Alshahrie et al., "Adsorption modeling and mechanistic insight of hazardous chromium on para toluene sulfonic acid immobilized-polyaniline@CNTs nanocomposites," Journal of Saudi Chemical Society, vol. 23, no. 2, pp. 188-197, 2019.

[39] Y. Xie, C. He, L. Liu et al., "Carbon nanotube based polymer nanocomposites: biomimic preparation and organic dye adsorption applications," RSC Advances, vol. 5, no. 100, pp. 82503-82512, 2015.

[40] K. Wu, J. Yu, and X. Jiang, "Multi-walled carbon nanotubes modified by polyaniline for the removal of alizarin yellow $r$ from aqueous solutions," Adsorption Science \& Technology, vol. 36, no. 1-2, pp. 198-214, 2018.

[41] P. Jayakaran, G. S. Nirmala, and L. Govindarajan, "Qualitative and quantitative analysis of graphene-based adsorbents in wastewater treatment," International Journal of Chemical Engineering, vol. 2019, Article ID 9872502, 17 pages, 2019.

[42] K. Cao, Z. Jiang, J. Zhao et al., "Enhanced water permeation through sodium alginate membranes by incorporating graphene oxides," Journal of Membrane Science, vol. 469, pp. 272-283, 2014.

[43] L. Dong, W. Fan, X. Tong, H. Zhang, M. Chen, and Y. Zhao, "A CO2-responsive graphene oxide/polymer composite nanofiltration membrane for water purification," Journal of Materials Chemistry A, vol. 6, no. 16, pp. 6785-6791, 2018.

[44] S. Kim, X. Lin, R. Ou et al., "Highly crosslinked, chlorine tolerant polymer network entwined graphene oxide membrane for water desalination," Journal of Materials Chemistry A, vol. 5, no. 4, pp. 1533-1540, 2017.

[45] N. Liu, M. Zhang, W. Zhang et al., "Ultralight free-standing reduced graphene oxide membranes for oil-in-water emulsion separation," Journal of Materials Chemistry A, vol. 3, no. 40, pp. 20113-20117, 2015.

[46] J. Zhao, H. Chen, H. Ye, B. Zhang, and L. Xu, "Poly (dimethylsiloxane)/graphene oxide composite sponge: a robust and reusable adsorbent for efficient oil/water separation," Soft Matter, vol. 15, no. 45, pp. 9224-9232, 2019.

[47] H.-Y. Mi, X. Jing, A. L. Politowicz, E. Chen, H.-X. Huang, and L.-S. Turng, "Highly compressible ultra-light anisotropic cellulose/graphene aerogel fabricated by bidirectional freeze drying for selective oil absorption," Carbon, vol. 132, pp. 199-209, 2018.

[48] L. P. Terrazas-Bandala, G. G. Sanchez, R. G. Valls et al., "Influence of humidity, temperature, and the addition of activated carbon on the preparation of cellulose acetate membranes and their ability to remove arsenic from water," Journal of Applied Polymer Science, vol. 131, no. 8, 2014.

[49] A. A. Alghamdi, A.-B. Al-Odayni, W. S. Saeed, A. Al-Kahtani, F. A. Alharthi, and T. Aouak, "Efficient adsorption of lead (II) from aqueous phase solutions using polypyrrole-based activated carbon," Materials, vol. 12, no. 12, p. 2020, 2019.

[50] I. Gardi and Y. G. Mishael, "Designing a regenerable stimuliresponsive grafted polymer-clay sorbent for filtration of water pollutants," Science and Technology of Advanced Materials, vol. 19, no. 1, pp. 588-598, 2018.

[51] A. M. Atta, H. A. Al-Lohedan, Z. A. AlOthman, A. A. AbdelKhalek, and A. M. Tawfeek, "Characterization of reactive amphiphilic montmorillonite nanogels and its application for removal of toxic cationic dye and heavy metals water pollutants," Journal of Industrial and Engineering Chemistry, vol. 31, pp. 374-384, 2015.

[52] H. Zeng, L. Wang, D. Zhang, F. Wang, V. K. Sharma, and C. Wang, "Amido-functionalized carboxymethyl chitosan/ montmorillonite composite for highly efficient and cost-effective mercury removal from aqueous solution," Journal of Colloid and Interface Science, vol. 554, pp. 479-487, 2019.

[53] F. Medhat Bojnourd and M. Pakizeh, "Preparation and characterization of a nanoclay/PVA/PSf nanocomposite membrane for removal of pharmaceuticals from water," Applied Clay Science, vol. 162, pp. 326-338, 2018.

[54] M. T. Nakhjiri, G. Bagheri Marandi, and M. Kurdtabar, "Effect of bis [2-(methacryloyloxy)ethyl] phosphate as a crosslinker on poly (AAm-co-AMPS)/Na-MMT hydrogel nanocomposite as potential adsorbent for dyes: kinetic, isotherm and thermodynamic study," Journal of Polymer Research, vol. 25, no. 11, p. 244, 2018.

[55] Y. Wang, Y. Xiong, J. Wang, and X. Zhang, "Ultrasonicassisted fabrication of montmorillonite-lignin hybrid hydrogel: highly efficient swelling behaviors and super- 
sorbent for dye removal from wastewater," Colloids and Surfaces A: Physicochemical and Engineering Aspects, vol. 520, pp. 903-913, 2017.

[56] J. Dou, Q. Huang, H. Huang et al., "Mussel-inspired preparation of layered double hydroxides based polymer composites for removal of copper ions," Journal of Colloid and Interface Science, vol. 533, pp. 416-427, 2019.

[57] X.-J. Yang, P. Zhang, P. Li et al., "Layered double hydroxide/ polyacrylamide nanocomposite hydrogels: green preparation, rheology and application in methyl orange removal from aqueous solution," Journal of Molecular Liquids, vol. 280, pp. 128-134, 2019.

[58] B. Nayak, A. Samant, R. Patel, and P. K. Misra, "Comprehensive understanding of the kinetics and mechanism of fluoride removal over a potent nanocrystalline hydroxyapatite surface," ACS Omega, vol. 2, no. 11, pp. 8118-8128, 2017.

[59] F. Zhao, E. Repo, D. Yin, Y. Meng, S. Jafari, and M. Sillanpää, "EDTA-Cross-Linked $\beta$-cyclodextrin: an environmentally friendly bifunctional adsorbent for simultaneous adsorption of metals and cationic dyes," Environmental Science \& Technology, vol. 49, no. 17, pp. 10570-10580, 2015.

[60] M. N. Sahmoune, "Thermodynamic properties of heavy metals ions adsorption by green adsorbents," in Green Adsorbents for Pollutant Removal: Fundamentals and Design, G. Crini and E. Lichtfouse, Eds., Springer, Cham, Switzerland, pp. 193-213, 2018.

[61] H. N. Tran and H.-P. Chao, "Adsorption and desorption of potentially toxic metals on modified biosorbents through new green grafting process," Environmental Science and Pollution Research, vol. 25, no. 13, pp. 12808-12820, 2018.

[62] K. L. Tan and B. H. Hameed, "Insight into the adsorption kinetics models for the removal of contaminants from aqueous solutions," Journal of the Taiwan Institute of Chemical Engineers, vol. 74, pp. 25-48, 2017.

[63] Y. Liu, X. Liu, W. Dong, L. Zhang, Q. Kong, and W. Wang, "Efficient adsorption of sulfamethazine onto modified activated carbon: a plausible adsorption mechanism," Scientific Reports, vol. 7, no. 1, Article ID 12437, 2017. 